\title{
Investigations into the unsteady internal flow characteristics for a waterjet propulsion system at different cruising speeds
}

\author{
Renfang Huang ${ }^{a}$, Weixiang Ye ${ }^{\text {b,e }}$, Yuanxing Dai ${ }^{c}$, Xianwu Luo ${ }^{\text {b,e }}$, Yiwei Wang ${ }^{\text {a,d, }}$, \\ Tezhuan Du ${ }^{\mathrm{a}, \mathrm{d}}$, Chenguang Huang ${ }^{\text {a,d }}$ \\ ${ }^{\text {a }}$ Key Laboratory for Mechanics in Fluid Solid Coupling Systems, Institute of Mechanics, Chinese Academy of Sciences, Beijing, 100190, China \\ ${ }^{\mathrm{b}}$ Department of Energy and Power Engineering, Tsinghua University, Beijing, 100084, China \\ ${ }^{\mathrm{c}}$ Science and Technology on Water Jet Propulsion Laboratory, Shanghai, 200011, China \\ ${ }^{\mathrm{d}}$ School of Engineering Science, University of Chinese Academy of Sciences, Beijing, 100049, China \\ ${ }^{\mathrm{e}}$ State Key Laboratory of Hydroscience and Engineering, Tsinghua University, Beijing, 100084, China
}

\section{A R T I C L E I N F O}

\section{Keywords:}

Waterjet

Waterjet propulsion

Pressure fluctuation

Numerical simulation

\begin{abstract}
A B S T R A C T
Unsteady turbulent flows in a waterjet propulsion system are investigated at various cruising speeds with the emphasis on pressure fluctuations. The numerical methodology is based on the Reynolds-Averaged Navier-Stokes (RANS) equation with the SST $k$ - $\omega$ turbulence model and a sliding mesh technique. The head and efficiency of the waterjet pump are predicted fairly well compared with the available experimental data. The pressure fluctuates intensively in the impeller and the dominant frequency is the impeller rotating frequency with the largest amplitude near the impeller inlet. Besides, two dominant frequency components exist in the intake duct and the diffuser. A high-frequency component is caused by the rotor-stator interaction, and another component is generated by the unsteady vortex evolution in the diffuser passage and would propagate upstream to the impeller and the intake duct. Analyses based on the vorticity transport equation demonstrate the great contribution of the vortex stretching term to the vorticity distribution and evolution in the diffuser. Finally, at the cruising speed of $45 \mathrm{knot}$, the flows inside the duct are strongly affected by the impeller rotation and present a periodic prewhirl motion with the dominant frequency of the impeller rotating frequency.
\end{abstract}

\section{Introduction}

Waterjet propulsion system is composed of the intake duct, the pump, the nozzle and the steering device. It pushes the marine vessels forward by using the reacting force of the waterjet pump, and controls the cruising direction by allocating and changing the jet flow direction through the steering device. The waterjet propulsion has many advantages, such as high propulsive efficiency, good maneuverability, and less vibration. Besides, the anti-cavitation performance is good since the static pressure at the impeller surface increases by diffusing the crosssectional area of the intake duct. Therefore, waterjet propulsion is widely used in both high-speed ferries and military amphibian vehicles (Park et al., 2005a, 2005b).

Due to its importance in a wide range of marine engineering applications, many efforts have been made in the past decades to predict the performance characteristics of the waterjet propulsion. Based on the momentum and energy flux method proposed by the 22nd International
Towing Tank Conference (ITTC) (ITTC, 1999), Bulten and van Esch (2005) predicted the thrust of a propulsion unit from a direct summation of all wall forces or from summation of the different terms of a momentum balance. Comparison of both methods shows that deviations for some operating conditions are attributed to the neglect of the pressure forces on the streamtube. Delaney et al. (2009) used the same method and also found that the predicted power was within $2 \%$ of the experimental values. Although the virtual streamtube was mentioned in literature (Bulten and van Esch, 2005; Delaney et al., 2009), they did not describe the specific solution. Instead, Ding and Wang (2010) introduced an additional User-Defined Scalar (UDS) equation embedded into Fluent to get the virtual streamtube surface between the flow ingested into the inlet duct and the external flow beneath the hull. Due to the limitations in the experiments, it is difficult to directly evaluate the waterjet propulsion performance in the towing tank. Therefore, an actuator disk model is used to replace a waterjet pump by Hino and Ohashi (2009) to numerically evaluate the performance of a waterjet

\footnotetext{
* Corresponding author.

E-mail addresses: hrenfang@yeah.net (R. Huang), wangyw@imech.ac.cn (Y. Wang).
} 
propelled ship. Altosole et al. (2012) proposed two original dimensionless numerical procedures for the selection and the performance prediction of the propulsion system at the early design stage of a high-speed craft. One procedure is based on a generalized performance map for mixed flow pumps and it is referred to jet units for naval applications. The other procedure is based on a complete physical approach and is more suitable for planning boats. Fluctuating noise sources of the full-scaled waterjet were numerically analyzed by hybrid method coupling Scale-Adaptive Simulation (SAS) with boundary element acoustic models (BEM) (Yang et al., 2014). Their results indicate that the pulsating pressure after the rotor and before the stator is the most-dominant second blades passing frequency (2BPF) and the most-dominated tonal noise at $2 \mathrm{BPF}$ is $136.2 \mathrm{~dB}$.

Furthermore, many investigations are conducted to illustrate the internal flow features and the effects on the performance characteristics. van Esch and Cheng (2011) experimentally and numerically studied the relation between the instability in the head curve and unsteady hydraulic forces. It is found that the instability of the head curve is not only connected with unsteady forces and rotating instabilities but also dependent on the clearance gap between impeller blades and casing. Gao et al. (2008) comprehensively investigated the flow fields at different flow rates, such as the pressure distribution on the blade surfaces, and the axial and tangential velocity distribution, especially the radial loading distribution. He further analyzed the effects of a rear stator and different spacing between the rotor and the stator on the overall performance and the flow fields. Olsson (2008) numerically simulated the cavitating flows in a waterjet system with different cavitation models, and the cavitation model proposed by Sauer and Schnerr (2000) showed good agreement with the experimental data. A barotropic state law is proposed by Pouffary et al. (2008) to simulate the cavitation phenomenon in turbomachinery and the results are in good accordance with available experimental data. Based on the relative vorticity transport equation, Huang et al. (2015a) analyzed the cavitation-vortex interaction in a mixed-flow waterjet pump. It is demonstrated that vortex dilation and baroclinic torque exhibit a steep jump as cavitation occurs and the vortex stretching contributes to large-scale vortex generation. The stall behavior of a mixed-flow waterjet pump was numerically studied by Hu et al. (2014) using the realizable $k-\varepsilon$ turbulence model. Results show that the performance curve presents a positive slope at $28-59 \%$ of the designed flow rate due to the stall flow in the impeller channels rather than the reverse flow in the diffuser. The stall flow is observed at the leading edge of the blade tip with developing from the blade suction surface (SS) to pressure surface (PS), and the stall region increases gradually from three to five impeller passages with decreasing flow rate. Cao et al. (2017) analyzed the performance deviation between the uniform and non-uniform suction flows, and found that the large non-uniform suction flows would cause a substantial drop in the pump head with the primary feature of a distinct swirl distortion near the top evolving into a circumferential vortex.

Although many interesting studies have been reported on the internal flow characteristics as mentioned above, they are not yet fully understood due to the complex features of the tip leakage vortex (Cheng et al., 2020; Guo et al., 2019). Lindau et al. (2012) numerically studied the cavitating flow in an axial flow waterjet propulsion over a wide range of flow coefficients. Results showed that the captured suction-surface and tip-gap cavitation patterns were blade-passage steady and periodic as observed in experiments. The three-dimensional flow structures and turbulence within the tip leakage vortex (TLV) of an axial waterjet pump were extensively measured by using the Stereo particle image velocimetry (SPIV) technique (Miorini et al., 2010, 2012; Wu et al., 2011a, 2011b). The results show that TLV starts to roll up along the suction surface (SS) tip corner of the blade and then propagates within the passage toward the pressure surface (PS) of the neighboring blade. Meanwhile, the TLV involves entrainment of a few vortex filaments with predominantly circumferential vorticity from the blade tip, and these filaments appear as swirling jets after shedding from the blade. Subsequently, Tan et al. investigated the effect of large scale cavitating vortical structures on the performance breakdown of an axial waterjet pump by using high-speed imaging as well as pressure and performance measurements (Tan et al., 2012, 2015). The results indicate that the interaction between the tip leakage vortex (TLV) and trailing edge of the attached cavitation near the rotor blade tip covering the suction surface plays a key role in performance breakdown, and the vortical cloud cavitation developing at the trailing edge of the sheet cavity near the blade tip is re-oriented by the TLV in a direction that is nearly perpendicular to the SS surface. Similar large perpendicular vortical structures have been observed in the heavily loaded cavitating rocket inducers by Acosta (1958).

The waterjet propulsion system is installed in one marine vessel, so it is necessary to understand the physics behind the waterjet/hull interaction (Gong et al., 2019). Eslamdoost devoted himself to this topic and proposed a validated numerical technique for computing the hydrodynamics of waterjet-driven hulls (Eslamdoost, 2012, 2014). The algorithm in the first part is called the pressure jump method (Eslamdoost et al., 2014). This method is based on the equilibrium condition that the resistance forces are balanced by the thrust force and coupled with a potential flow solver to simulate the sinkage and trim changes. In the second part, a technique using a RANS solver with a Volume of Fluid (VOF) combined with a body force representation of the pump is developed and validated against measurements (Eslamdoost et al., 2018). Based on the results, the transom clearance is found to play an important role in the behavior of the thrust deduction fraction in the low speed range. In contrast, Forest et al. (2012) used a two degree-of-freedom (DOF) unsteady model to investigate the equilibrium of the boat at steady speed and mimic the system dynamics. The numerical model includes a planning hull with an inlet duct and a mathematical representation of the main forces and mass flow to model the pump.

Although the performance characteristics and internal flow features have been studied, a brief review of these recent works indicates that we still have inadequate understanding on the pressure fluctuations and vortex evolutions in a waterjet propulsion system at different cruising speeds, since it is difficult to measure the pressure at the impeller and visualize the transient flow patterns. The objective of this work is to shed light on the unsteady internal flow characteristics of a waterjet propulsion system at different cruising speeds with the emphasis on the pressure fluctuations.

\section{Numerical simulation}

\subsection{Computational domain and boundary conditions}

The three-dimensional computational domain is shown in Fig. 1. The waterjet propulsion system is composed of a water tank, an intake duct, a mixed-flow impeller, a diffuser and a nozzle. In order to consider the effect of the hull boundary layer, the water tank is $10 D$ wide and $8 D$ high, the inlet plane is $25 D$ in front of the intake duct, and the outlet plane is $5 D$ behind the intake duct, where $D=244 \mathrm{~mm}$ is the duct diameter. This is recommended by Liu et al. (2010).

Note that there are six blades in the mixed-flow impeller and eleven blades in the diffuser. The geometrical parameters are described in the literature (Huang et al., 2019b) with the meridional schematic diagram for the waterjet pump shown in Fig. 2. The geometrical parameters are listed in Table 1 . The blade width ratio at the inlet is 0.318 , the blade width ratio at the exit is 0.247 , the blade inlet diameter at the tip is 0.856 , the blade inlet diameter at the hub is 0.245 and the blade exit diameter at the hub is 0.604 with the blade outlet diameter at shroud of $D_{2}=345 \mathrm{~mm}$.

The unsteady turbulent flows are simulated using the CFD code ANSYS-CFX based on the Reynolds-averaged Navier-Stokes (RANS) equation with the SST $k$ - $\omega$ turbulence model, which is evaluated via many benchmark cases by Bardina et al. (1997). This turbulence model 


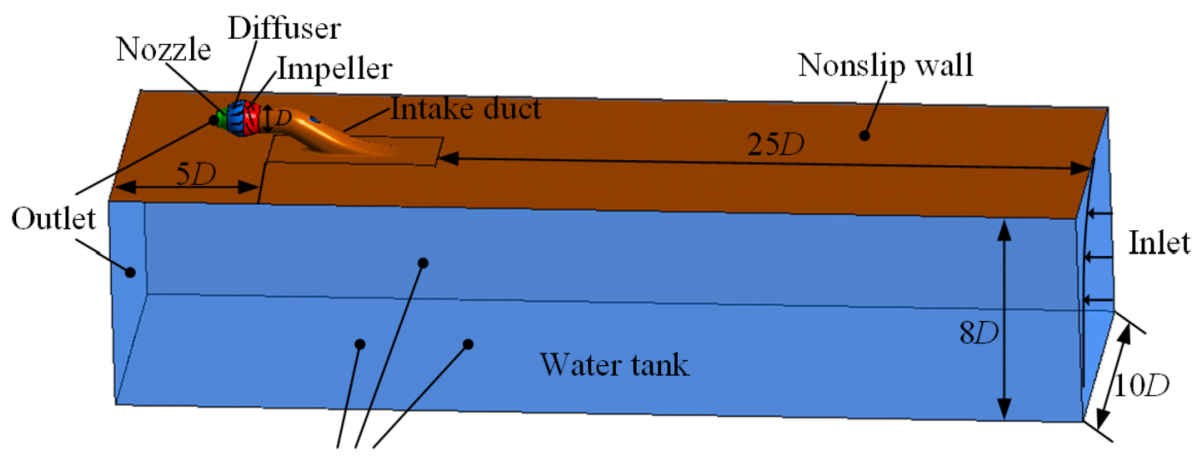

Free slip wall

Fig. 1. Computational domain and boundary conditions of the waterjet propulsion system (Luo et al., 2020).

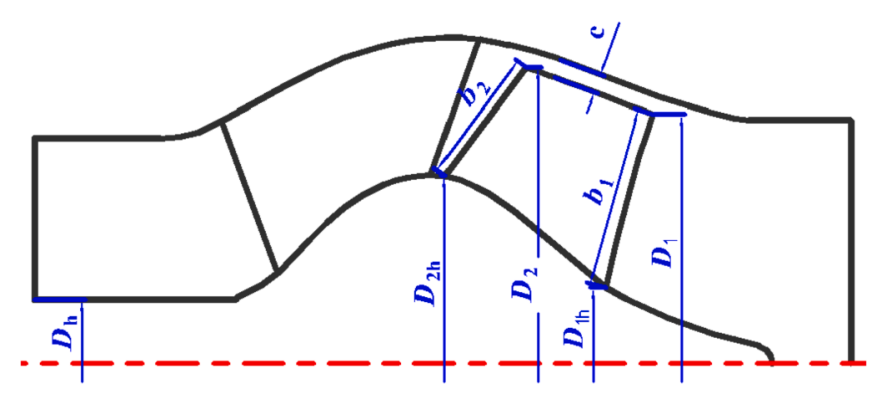

Fig. 2. Meridional schematic diagram for the pump (Huang et al., 2019b).

Table 1

Geometrical parameters for the impeller (Huang et al., 2019b).

\begin{tabular}{lll}
\hline Parameters & Symbols & Values \\
\hline Blade width ratio at the inlet & $b_{1} / D_{2}$ & 0.318 \\
Blade width ratio at the exit & $b_{2} / D_{2}$ & 0.247 \\
Blade inlet diameter at the tip & $D_{1} / D_{2}$ & 0.856 \\
Blade inlet diameter at the hub & $D_{1 \mathrm{~h}} / D_{2}$ & 0.245 \\
Blade exit diameter at the hub & $D_{2 \mathrm{~h}} / D_{2}$ & 0.604 \\
Hub ratio & $D_{\mathrm{h}} / D_{2}$ & 0.209 \\
Blade tip clearance & $c / D_{2}$ & $0.86 \mathrm{e}-3$ \\
\hline
\end{tabular}

shows suitable performance to predict flow separations under adverse pressure gradients and flow structures around the rotating blades (Ji et al., 2012; Liu et al., 2019; Liu and Tan, 2019). Water at $25^{\circ} \mathrm{C}$ is used as the flow medium, whose density is $997 \mathrm{~kg} / \mathrm{m}^{3}$ and dynamic viscosity is $8.899 \times 10^{-4} \mathrm{~kg} \cdot \mathrm{m}^{-1} \cdot \mathrm{s}^{-1}$. A rotational coordinate system is set in the impeller, a stationary coordinate system is set in the other components, and thus the sliding mesh technique is adopted at the interfaces between the two reference systems. The time-dependent governing equations are discretized both in space and time using the direct coupling method (Vanka, 1986). The high order resolution scheme is used for the convection terms with the central difference scheme used for the diffusion terms in the governing equations. The unsteady second-order backward Euler formation is used for the transient term. For the convergence control, maximum iteration for each physical time step is 10 until the RMS (root mean square) residual drops below $10^{-5}$. During the unsteady calculation, the time step corresponds to $1^{\circ}$ per step.

The boundary conditions are set as follows. For the inlet plane of the water tank, a non-uniform velocity profile is used to simulate the development of the hull boundary layer and the definition is in Equation (1) (Bulten, 2006), where $V_{\mathrm{wt}}$ is the local velocity at the inlet plane of the water tank with a distance of $y_{\text {rel }}$ from the hull, $V_{s}$ is the ship cruising speed, $\delta$ is the thickness of the hull boundary layer, $L_{\text {in }}$ is the distance from the domain inlet plane to the inlet, $L_{\mathrm{in}}=25 D$, Re is Reynolds number, $\operatorname{Re}=V_{s} L_{\text {in }} / v, v$ is the fluid kinematic viscosity. For the outlet planes, the average static pressure is set as a constant value. It is noted that the average static pressure is constant but the pressure together with the velocity at the nozzle exit are allowed to be non-uninform distributions, which is in accordance with the results demonstrated by Eslamdoost and Vikström (2019). Free slip wall boundary condition is applied at the bottom plane and the side planes of the water tank, and therefore the mesh near these walls can be made relative coarse without resolving the boundary layer. The nonslip wall boundary is applied at the other solid walls.

$$
\begin{aligned}
& \left\{\begin{array}{l}
V_{\mathrm{wt}}=V_{\mathrm{s}}\left(y_{\mathrm{rel}} / \delta\right)^{1 / 9} \\
V_{\mathrm{wt}}=V_{\mathrm{s}}, y_{\mathrm{rel}}>\delta
\end{array}, y_{\text {rel }} \leq \delta\right. \\
& \delta=0.27 L_{\text {in }} \bullet \mathrm{Re}^{-1 / 6}
\end{aligned}
$$

Fig. 3 shows the navigational characteristics of the waterjet propulsion system. Three operation conditions (marked with blue diamonds) are selected to study in this paper with the input power of $310.4 \mathrm{~kW}$ and the cruising speed of $34,40,45 \mathrm{knot}$, corresponding to the rotation speed of $2809,2818,2829 \mathrm{rpm}$, respectively. Although the shaft delivered power is the same, the propulsion thrust gradually decreases along with the increase in the cruising speed. It is noted that the resistance and the thrust are not in balance at these three operation conditions since the internal flow characteristics at transient states are of the major interest in this paper rather than that at the balanced states. All the calculations are conducted on servers with 12 Intel Xeon X5670 core processors and a 160G hard drive, which are supported by Tsinghua National Laboratory for Information Science and Technology.

\subsection{Mesh generation}

Hybrid mesh is generated and employed in present simulation. The structured grids are generated for the impeller and diffuser components by the commercial codes ANSYS TurboGrid 16.0, and the other component mesh is generated by the commercial codes ANSYS ICEM 16.0. Mesh distribution for each component is shown in Fig. 4. As shown in Fig. 4(b), the unstructured grids are used in the intake duct. In order to meet the requirement of the turbulence model, the tetrahedral grids exist in the majority of the intake duct, and the prism grids are adopted near the boundary with the largest $y+$ below 50 . As shown in Fig. 4(d) and (e), O-H type grids are produced in the impeller and diffuser, and hence sufficient refinement can be made around the blade surfaces to capture flow features inside boundary layers.

The grid sensitivity is performed by using three mesh resolutions with the grid refinement ratio of $r=1.3$ in $x, y, z$ directions. Several monitoring points are selected in the intake duct, the impeller blade and the diffuser blade, as shown in Fig. 5. Note that points PI1 PI4 are set on the pressure side of the impeller blade, which are set spatially equally along the mid-chord curve. The points PV1 PV4 are set on the pressure side of the diffuser blade, which are also identically placed along the mid-chord curve. Especially, the PI1 PI4 are set in the relative 


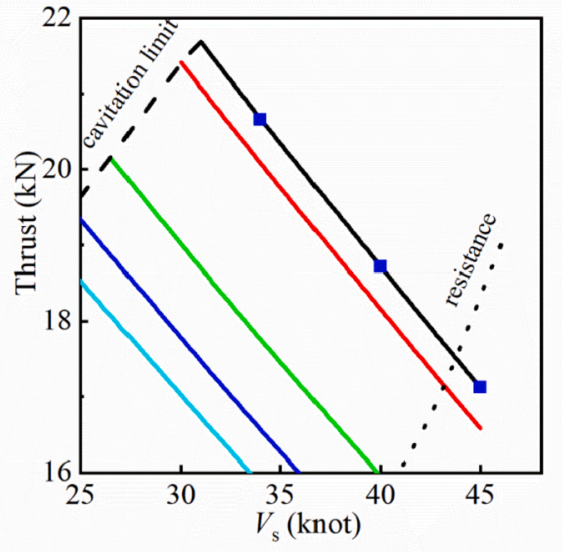

(a)

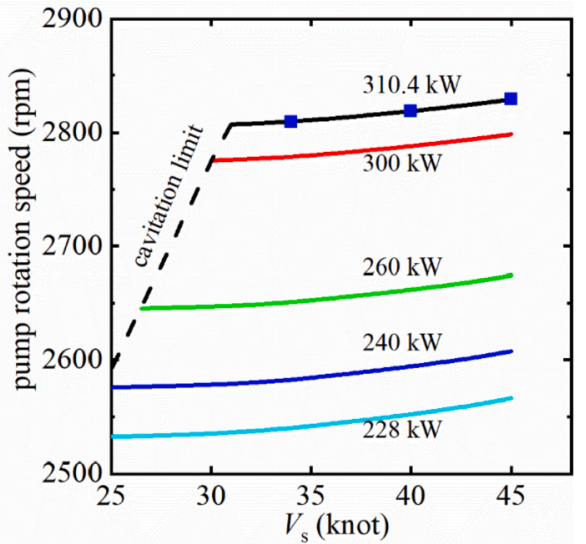

(b)

Fig. 3. Navigational characteristics of the waterjet propulsion system and present investigation conditions marked with blue diamonds.

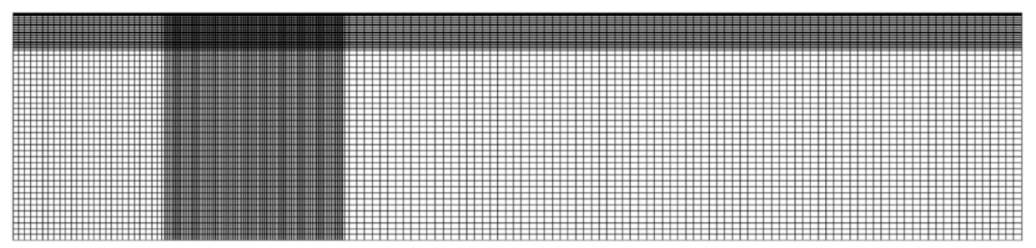

(a) Water tank
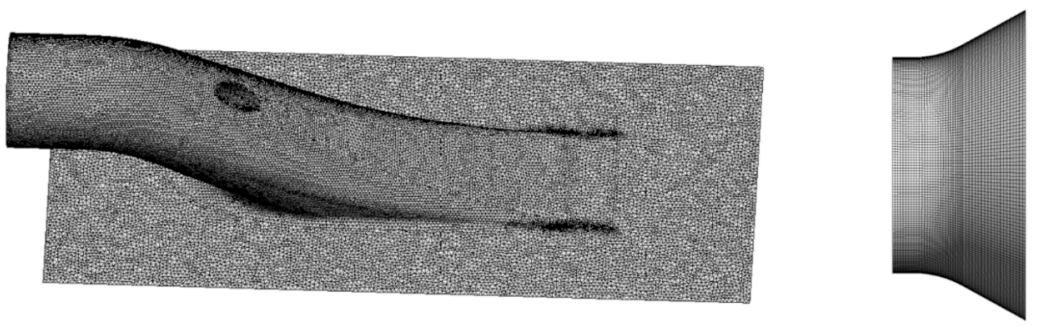

(b) Intake duct

(c) Nozzle

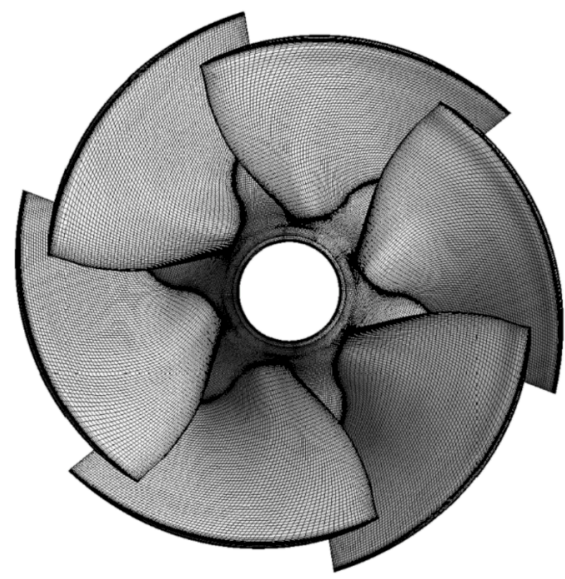

(d) Impeller

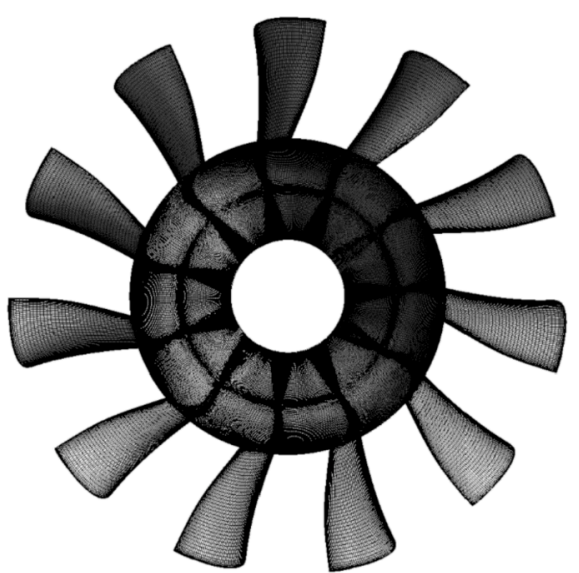

(e) Diffuser

Fig. 4. Mesh distribution for (a) water tank, (b) intake duct, (c) nozzle, (d) impeller and (e) diffuser.

coordinate system, which rotate along with the impeller blade. The grid convergence index (GCI) (Roache, 1993) is introduced to estimate the numerical uncertainty, which is currently the most reliable and recommended method that has been evaluated over several hundred numerical simulations (Eça et al., 2005, 2007; Huang et al., 2020). As shown in Table 2, the absolute pressure at those monitoring points shows that the uncertainty estimated by GCI method has a value less than $5 \%$, which demonstrates that the present simulation results are independent of the grid resolution. It should also be noted that further grid refinement leads to relatively more complex physics prediction but also causes an increasing computational cost together with the instability of the numerical simulation. Therefore, case 2 is selected as the 


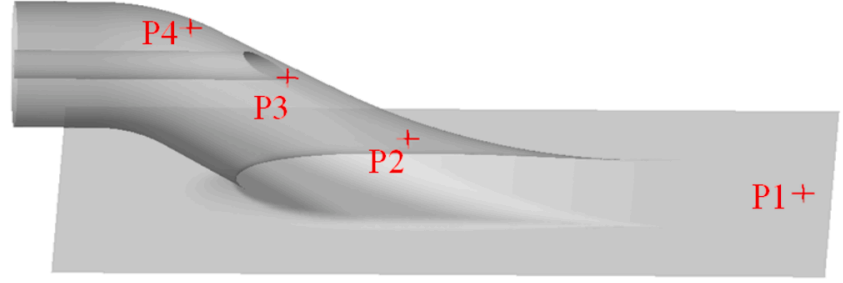

(a) Intake duct

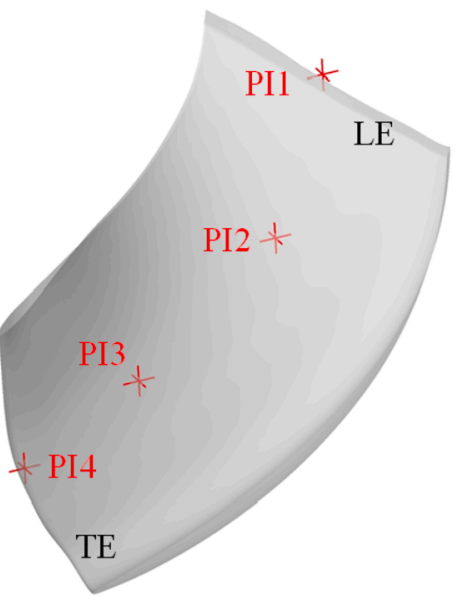

(b) Impeller

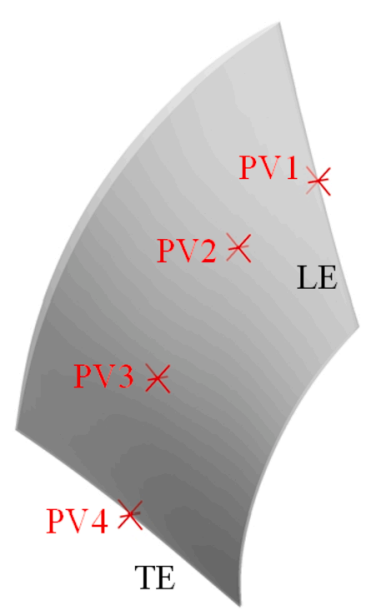

(c) Diffuser
Fig. 5. Location diagram of monitoring points (a) intake duct, (b) impeller blade, and (c) diffuser blade.

final mesh and the final grid elements are 14 million.

\subsection{Numerical method validation}

Comparisons of the waterjet pump performance between experiments and simulations are conducted in order to demonstrate the accuracy of numerical simulation in present study. Experimental measurements are conducted in the laboratory of the Marine Design and Research Institute of China. The model experimental device is shown in Fig. 6, including the straight inlet pipe, the impeller, the diffuser and the straight outlet pipe. The electromagnetic flow meter (with the uncertainty of $0.1 \%$ ) is installed upstream the impeller to measure the flow rate $(Q)$. The input power $(T)$ is acquired by a torque transducer (with the uncertainty of $0.2 \%$ ). One pressure sensor (with the uncertainty of $0.2 \%$ ) is installed upstream the pump with another pressure sensor (with the uncertainty of $0.2 \%$ ) installed downstream the pump. Therefore, the pump head $(H)$ and efficiency $(\eta)$ can be derived from the total pressure difference obtained by the two pressure sensors, and the formulations are as follows,

$H=\frac{p_{\mathrm{t} 2}-p_{\mathrm{t} 1}}{\rho g}$

$\eta=\frac{\rho g Q H}{T \cdot \omega}=\frac{Q\left(p_{\mathrm{t} 2}-p_{\mathrm{t} 1}\right)}{T \cdot \omega}$ where $p_{\mathrm{t} 1}, p_{\mathrm{t} 2}$ is the total pressure measured at the upstream and downstream of the pump, $T$ is the torque measured by a torque transducer, and $\omega$ is the angular speed of the impeller.

In order to validate the accuracy of numerical simulation in present study, the total pressure should be obtained at the same locations between simulations and experiments. Therefore, the present computation domain is made based on the experimental model, including the inlet pipe, the impeller, the diffuser and the outlet pipe. Two monitoring planes are assigned at the same locations where the pressure sensors are installed in experiments, and then the corresponding total pressures can be calculated. Besides, the torque is the integral torque from the rotating component based on the simulation results.

The experimental performance (marked as "exp.") and simulation data (marked as "cal.") are presented in Fig. 7. The calculated head is in good agreement with the experimental head. The calculated torque is lower than the experimental torque at the same flow rate, causing the calculated efficiency is higher than the experimental efficiency, but both the efficiency and the torque exhibit the same tendency between the experiments and the simulations. The discrepancy of torque between calculation and experiment is due to the mechanical torque, which is not included in calculation. The discrepancy in efficiency is attributed to two factors (Huang et al., 2015b). Firstly, the efficiency calculated based on numerical simulation is the hydraulic efficiency, but the efficiency measured from experiments includes the effects of the mechanical and volumetric losses of the pump. Additionally, due to the processing deviations, especially at the leading edge, the simulated torque does not match well with the experiments as shown in Fig. 7 (b). Note that the maximum efficiency deviation is $1.4 \%$ at the off-design flow rate of $Q=$ $0.35 \mathrm{~m}^{3} / \mathrm{s}$. Therefore, this numerical simulation method is reliable to predict the hydrodynamic performance, and the following study will focus on the transient characteristics inside the waterjet propulsion system at different conditions.

\section{Results and discussions}

\subsection{Performance comparisons}

Performances of the intake duct and the waterjet pump are analyzed and discussed at three cruising speeds.

The performance evaluation indexes for an intake duct are hydraulic

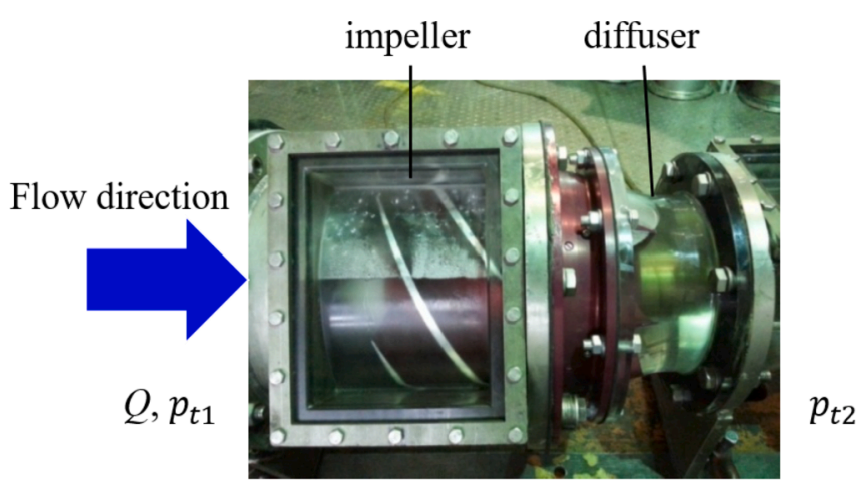

Fig. 6. Model experimental device (Li et al., 2017).

Table 2

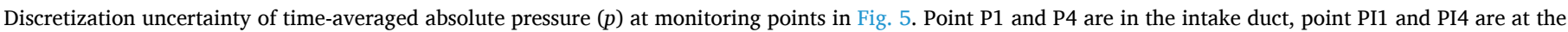
impeller blade, point PV1, PV3 and PV4 are at the diffuser blade. The unit for the absolute pressure is Pa.

\begin{tabular}{|c|c|c|c|c|c|c|c|c|}
\hline Mesh & Elements & P1 & P4 & PI1 & PI4 & PV1 & PV3 & PV4 \\
\hline Case 1 & $24,280,095$ & -9899 & 83289 & 449107 & 330399 & 591687 & 358776 & 439294 \\
\hline Case 2 & $14,282,409$ & -9861 & 83674 & 456013 & 342590 & 601205 & 359078 & 440840 \\
\hline Case 3 & $8,401,417$ & -9796 & 84210 & 469095 & 346928 & 609058 & 360514 & 442899 \\
\hline GCI & 1 & $0.93 \%$ & $1.47 \%$ & $2.15 \%$ & $2.23 \%$ & $3.03 \%$ & $2.88 \%$ & $1.37 \%$ \\
\hline
\end{tabular}




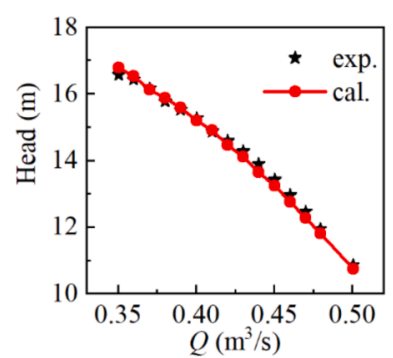

(a)

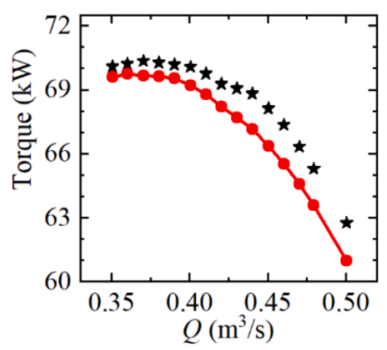

(b)

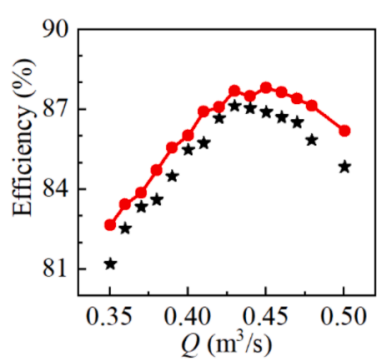

(c)

Fig. 7. Comparison of experimental and numerical results.

efficiency $\eta_{\text {duct }}$, outflow nonuniformity $\xi$ and perpendicularity $\varphi_{\mathrm{p}}$, which are defined in Equation (3) (5), where $E_{\text {in }}$ is the total energy at the inlet plane, $E_{\text {out }}$ is the total energy at the outlet plane, $\rho$ is fluid density, $V_{\text {in }}$ and $V_{\text {out }}$ is the velocity magnitude at the inlet plane and outlet plane, respectively, $p_{\text {in }}$ and $p_{\text {out }}$ is the static pressure at the inlet plane and outlet plane, respectively, $p_{\mathrm{r}}$ is the reference pressure, $V_{\mathrm{a}}, \overline{V_{\mathrm{a}}}$ and $V_{\mathrm{t}}$ is the axial velocity, the averaged axial velocity and the tangential velocity at the outlet plane, respectively. Note that the subscript "inlet" means the capture area positioned one impeller diameter forward of the ramp tangent point, the subscript "outlet" means the duct outlet plane which is also the interface between the impeller and the duct. It is well known that there is a stream tube separating the ingested water from the external water in the water tank. Besides, the capture area has a slight effect on the calculation of the duct hydraulic efficiency and can be described by a semi-ellipse according to the investigations by Griffith-Jones (1994). Therefore, the capture area (i.e. the duct inlet area) is defined by a semi-ellipse which satisfies the mass conservation as shown in Fig. 8.

$\eta_{\text {duct }}=\frac{E_{\text {out }}}{E_{\text {in }}}=\frac{\int\left[0.5 \bullet \rho V_{\text {out }}^{2}+\left(p_{\text {out }}-p_{\mathrm{r}}\right)\right] d Q}{\int\left[0.5 \bullet \rho V_{\text {in }}^{2}+\left(p_{\text {in }}-p_{\mathrm{r}}\right)\right] d Q}$

$\xi=\frac{1}{Q} \int_{d A}\left|V_{\mathrm{a}}-\overline{V_{\mathrm{a}}}\right| d A$

$\varphi_{\mathrm{p}}=\frac{1}{Q} \int_{d A} V_{\mathrm{a}}\left[90^{\circ}-\arctan \left(\frac{V_{\mathrm{t}}}{V_{\mathrm{a}}}\right)\right] d A$

Fig. 9 shows the performance comparisons for the intake duct at three cruising speeds with evaluation indexes, i.e. hydraulic efficiency $\eta_{\text {duct }}$ outflow nonuniformity $\xi$ and perpendicularity $\varphi_{\mathrm{p}}$. Note that $\xi$ is

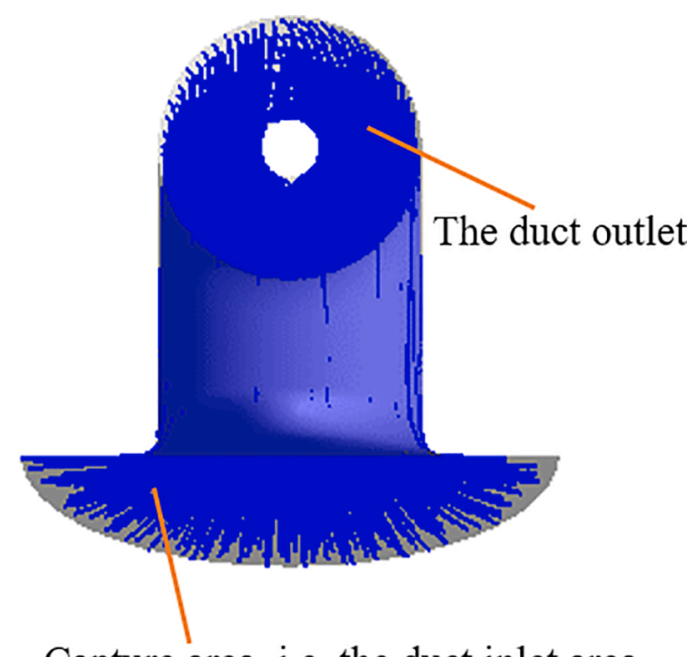

Capture area, i.e. the duct inlet area

Fig. 8. The capture area and the duct outlet plane. amplified 100 times for better illustration. When increasing the cruising speed, the duct efficiency varies slightly with maximum deviation of $\Delta \eta_{\text {duct }}=0.88 \%$, the nonuniformity increases from $\xi=0.11$ to $\xi=0.15$, while the perpendicularity drops from $\varphi_{\mathrm{p}}=83.72^{\circ}$ to $\varphi_{\mathrm{p}}=82.69^{\circ}$. Since the perpendicularity $\varphi_{\mathrm{p}}$ represents the ratio of the flow rate in the axial direction to the total flow rate, a large $\varphi_{\mathrm{p}}$ value implies the flow rate in the axial direction plays a significant role in the main flows at the current plane. It is widely acknowledged that the lower the nonuniformity together with the larger the perpendicularity, so the better the outflow quality, which also demonstrates a better inflow condition provided for the waterjet pump. However, in this study, the outflow quality of the intake duct becomes worse along with the augment of the cruising speed.

In addition, the pump performances are further analyzed in Fig. 10. The flow rate of the waterjet pump ranges from $Q=0.61 \mathrm{~m}^{3} / \mathrm{s}$ to $Q=$ $0.65 \mathrm{~m}^{3} / \mathrm{s}$ along with the cruising speed increasing, and therefore the pump efficiency presents a monotonic decreasing trend with a reduction by $0.25 \%$, as depicted in Fig. 7 (c).

\subsection{Time-averaged flow features}

The nonuniformity $\xi$ and pressure distributions are used to analyse the time-averaged flow inside the waterjet propulsion system at three

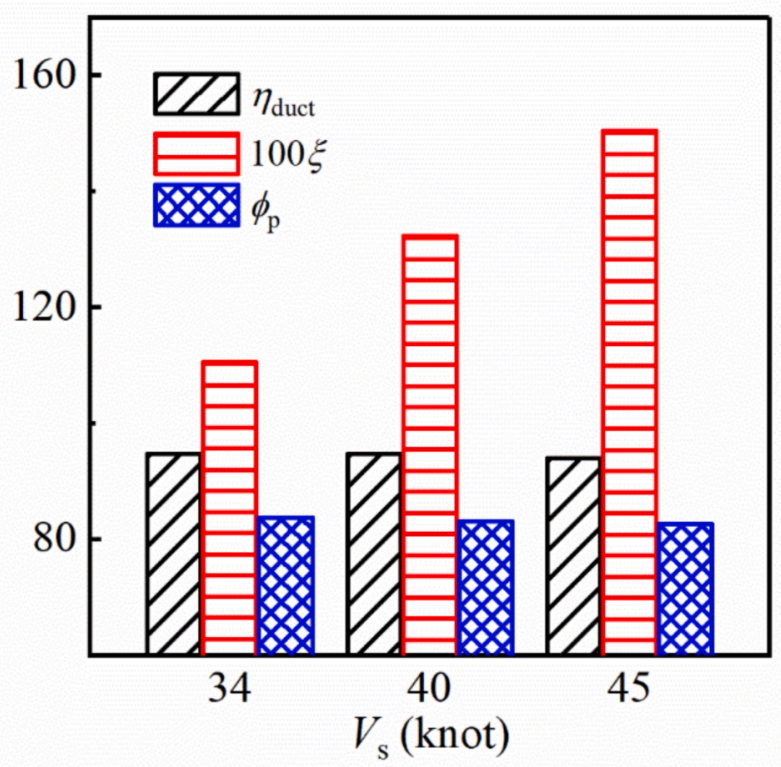

Fig. 9. Performance comparisons of the intake duct at three cruising speeds with evaluation indexes, i.e. hydraulic efficiency $\eta_{\text {duct }}$, outflow nonuniformity $\xi$ and perpendicularity $\varphi_{\mathrm{p}}$. Note that $\xi$ is amplified 100 times for better illustration. 
cruising speeds. Three typical planes are selected as shown in Fig. 11, where Plane 1 is located at the impeller inlet plane, Plane 2 is the interface plane between the impeller and the diffuser, and Plane 3 is the diffuser outlet plane.

Fig. 12 shows the nonuniformity $\xi$ comparisons at three measurement planes at three cruising speeds. The nonuniformity at Plane 2 is the largest, followed by Plane 3 and finally Plane 1. It is demonstrated that the uniform inflow becomes very chaotic after passing through the impeller, but the nonuniformity is improved to a large extent after the rectification of the diffuser. With increasing the cruising speed, the nonuniformity at Plane 1 increases from 0.11 to 0.15 with the grow rate of $36 \%$. It is because flows inside the intake duct become strongly turbulent with the increase of the cruising speed, and this leads to the nonuniformity increase at the Plane 1 , which will be clarified in section 3.3 by depicting the transient behaviors in the duct. Moreover, the nonuniformity at Plane 2 decreases from 0.42 to 0.38 with the reduction rate of $9.5 \%$, and the nonuniformity at Plane 3 decreases by $23 \%$ which ranges from 0.22 to 0.17 . This indicates the flow becomes uniform along with the increase of the cruising speed both in the impeller and diffuser. At the cruising speed of 45 knot, the velocity nonuniformity is alleviated to the most extent, especially in the diffuser, which will be also discussed in section 3.3.

Fig. 13 shows the time-averaged pressure distributions at three measurement planes. The pressure gradually increases when the flow passes through the impeller and then it drops slightly after the diffuser. For three planes, the pressure increases together with the cruising speed and it presents different pressure distribution characteristics. In Fig. 13 (a), the pressure is distributed unsymmetrically at the impeller inlet plane (i.e., Plane 1), that is, the pressure on the left side is lower than the pressure on the right side. Meanwhile, the velocity on the left side is larger than that on the right side as depicted in Fig. 14 (a), which is caused by the rotation effect of the waterjet impeller. In contrast, the velocity distribution in Fig. 14 (b) is symmetrical at Plane 1 without the rotation effect of the downstream impeller according to investigations in the literature (Huang et al., 2019a). As the flow moves to the interface plane between the impeller and the diffuser (i.e. Plane 2), the alternating presence of high pressure and low pressure is obviously observed in Fig. 13 (b) due to the rotor-stator interaction, and this will give rise to strong pressure pulsation and turbulent vortex evolution which will be analyzed in section 3.3. For the outlet plane of the diffuser (i.e., Plane 3), since the effect of the rotor-stator interaction gradually decreases as the flow propagates downstream, the pressure shows a similar distribution with that at Plane 2, but the alternating phenomena of high pressure and low pressure is not so apparent. In addition, the pressure alternating phenomenon becomes distinct along with the augment of the cruising speed.

The unsteady behaviours are further analyzed for the better

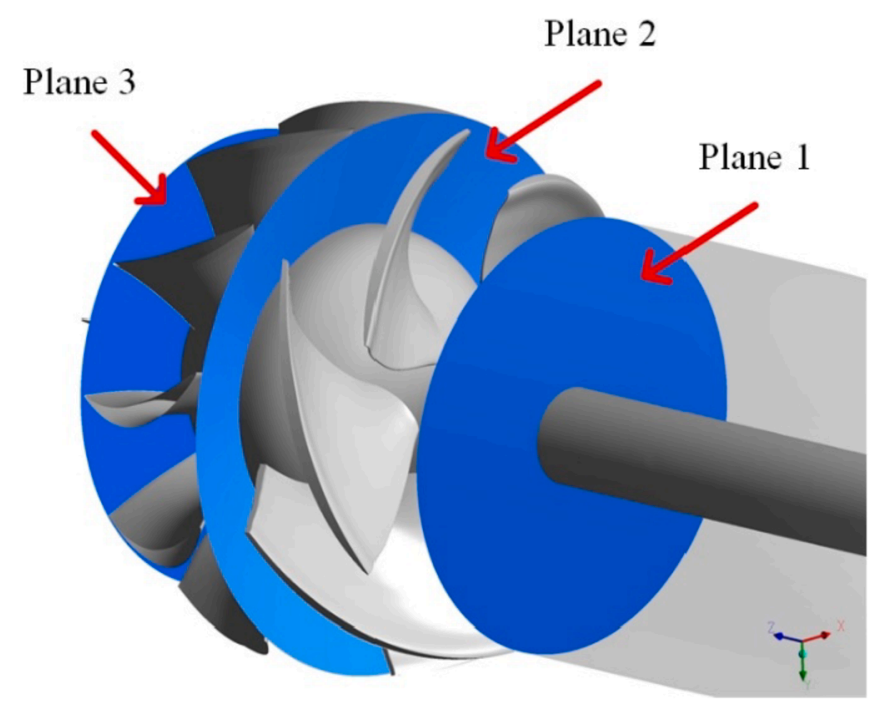

Fig. 11. Location diagram of three measurement planes.

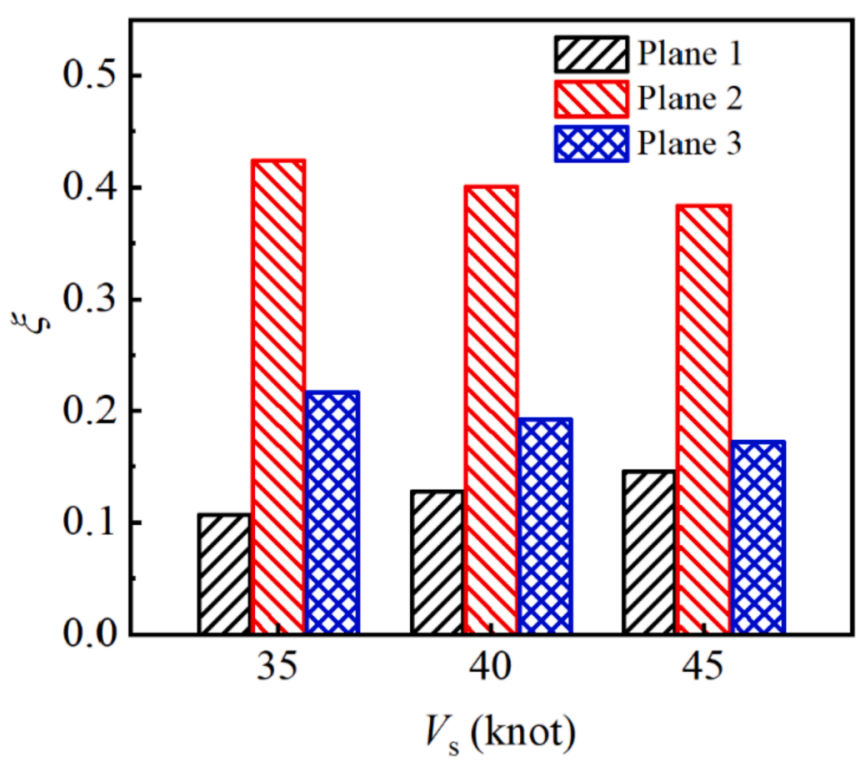

Fig. 12. Nonuniformity $\xi$ comparisons at three measurement planes at three cruising speeds.

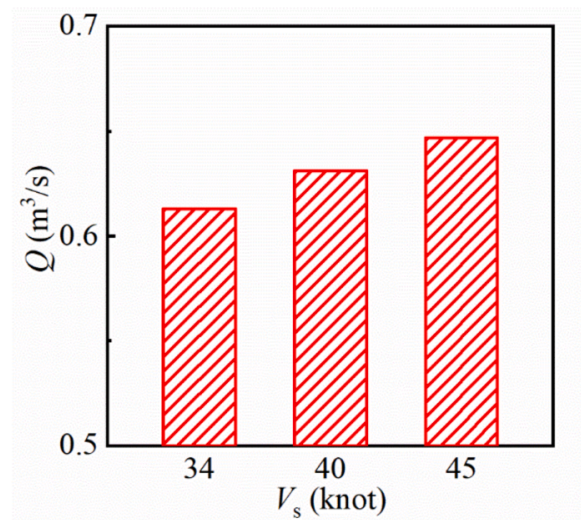

(a)

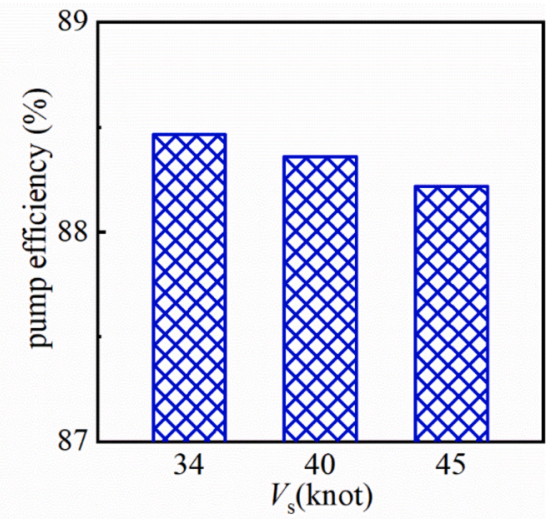

(b)

Fig. 10. Pump performance comparisons at three cruising speeds. 


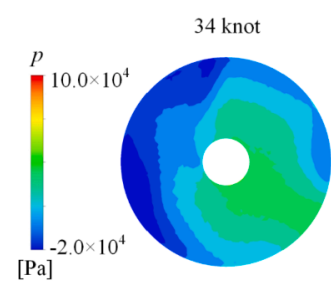

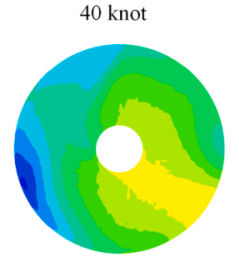

(a)
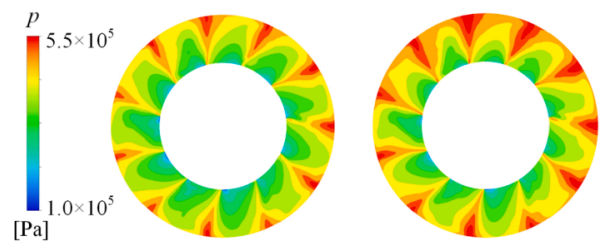

(b)
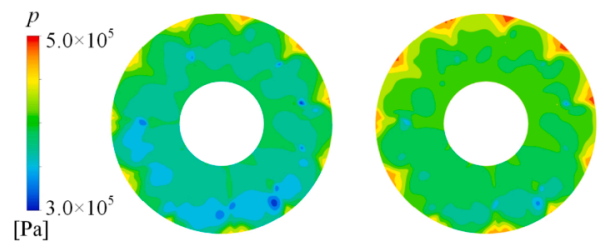

(c)
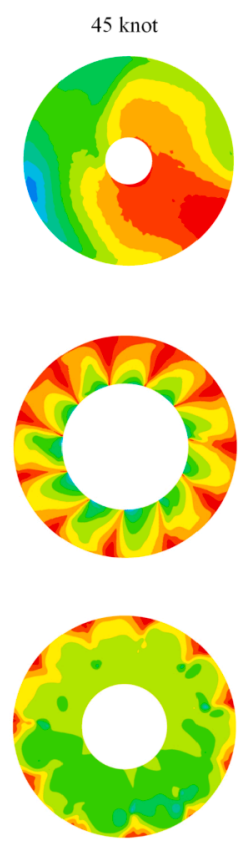

Fig. 13. Time-averaged pressure distributions at three measurement planes: (a) Plane1; (b) Plane2; (c) Plane3.

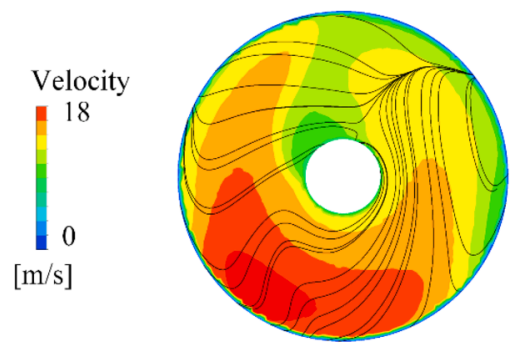

(a)

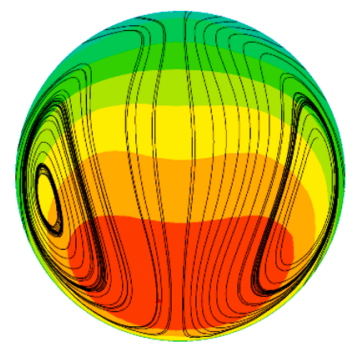

(b)
Fig. 14. Time-averaged velocity distributions at the Plane 1: (a) with the impeller; (b) without the impeller.

understanding of the transient internal flows of the propulsion system at different cruising speeds.

\subsection{Unsteady internal flows}

Since the pressure fluctuation induced by the complex internal flows in the waterjet propulsion system is a major source of the noise and the hull vibrations, it is of great significance to analyse the characteristics of pressure fluctuations at various cruising speeds. Several monitoring points are selected in the intake duct, the impeller blade and the diffuser blade, as shown in Fig. 5.

Besides, the pressure coefficient $c_{\mathrm{p}}$ is used to analyse the pressure fluctuations with the definition as follows,

$c_{\mathrm{p}}=\frac{p_{i}-p_{0}}{0.5 \rho V_{2}^{2}}$

where $p_{i}$ presents the instantaneous value of static pressure at a monitoring point, $p_{0}$ is the averaged pressure at the impeller inlet plane, $V_{2}$ is the circumferential velocity at the impeller exit, $V_{2}=2 \pi n R_{2} / 60$ with $R_{2}$ defined as the radius of the blade tip at the exit and $n$ defined as the rotation speed.

Current simulations for each case are performed for approximately 25 rotation cycles. Flow statistics are sampled about 25 rotation cycles with the initial flow data corresponding to about 10 cycles rejected. Fast Fourier Transform is taken for the following 15 cycles.

\subsubsection{Impeller}

In the impeller, four monitoring points (PI1 PI4) are selected to analyse the pressure fluctuations by performing Fast Fourier Transform for the 15-cycle data. Fig. 15 shows the frequency spectrums of four monitoring points at various cruising speeds. It is along the flow direction from point PI1 to point PI4. The frequency spectrum shows the similar distribution among various cruising speeds. For a specific cruising speed, the impeller rotating frequency $\left(f_{n}\right)$ together with its harmonic frequencies are clearly observed at point PI1, and the pressure amplitudes of the impeller rotating frequency $\left(f_{n}\right)$ and its harmonic frequencies decrease dramatically at PI2 PI4 along with the fluid moving downstream.

As the dominant frequency in the impeller is the impeller rotating frequency, the instantaneous distributions of the turbulent kinetic energy and pressure on the blade pressure surface are given in Fig. 16 and Fig. 17. It is seen that the distributions of the turbulent kinetic energy $(k)$ and pressure $(p)$ are different on the pressure surface when the blade rotates to different positions.

As shown in Fig. 16 (a), a high- $k$ region is mainly distributed at the blade tip and close to the blade leading edge (LE), and there is a high- $p$ region near the blade leading edge (LE) and a low- $p$ region near the blade trailing edge (TE). The high- $k$ region decreases at $t_{0}+1 / 6 T$ and then increases at $t_{0}+2 / 6 T$. From $t_{0}+3 / 6 T$ to $t_{0}+5 / 6 T$, the high- $k$ region gradually decreases, and the evolution cycle of the turbulent kinetic energy corresponds to the impeller rotation cycle $\left(T=1 / f_{\mathrm{n}}\right)$. With the impeller rotating, the high- $p$ region generally increases until $t_{0}+2 / 6 T$, and then decreases from $t_{0}+3 / 6 T$ to $t_{0}+5 / 6 T$. Meanwhile, the minimum pressure value also increases from $t_{0}$ to $t_{0}+3 / 6 T$, and subsequently the low- $p$ region presents again at $t_{0}+4 / 6 T$ and $t_{0}+5 / 6 T$. The pressure variation is strongly connected with the periodic inflows as shown in Fig. 18. Although the velocity distribution at Plane 1 presents larger values on the lower side and smaller values on the upper side during one cycle, the flow structures (named as A) would change periodically. Besides, the periodic inflows are extensively discussed in section 3.3 .2 by depicting the normalized helicity in the intake duct. Note that the pressure is varied in the same pace with the inflow from the intake duct with the evolution cycle corresponding to the impeller rotation cycle ( $T$ $\left.=1 / f_{\mathrm{n}}\right)$.

\subsubsection{Intake duct}

Fig. 19 shows the pressure fluctuations of points P1 P4 inside the intake duct at three cruising speeds. It is in the flow direction from point P1 to point P4. At the cruising speed of 34 knot and $40 \mathrm{knot}$, the dominant component of the pressure fluctuation is sixth impeller rotating frequency $\left(6 f_{n}\right)$ together with its harmonic frequencies $\left(12 f_{n}\right)$ at the impeller inlet plane, and it is caused by the interaction between the rotating impeller and the static intake duct. Some components are observed near the 1.5 times impeller rotating frequency $\left(1.5 f_{\mathrm{n}}\right)$, but these signals are not strong enough to constitute the dominant component at speeds of 34 knot and 40 knot. When the cruising speed reaches 45 knot, internal flows become more complex inside the intake duct. Meanwhile, the sixth impeller rotating frequency $\left(6 f_{n}\right)$ and its harmonic frequency $\left(12 f_{\mathrm{n}}\right.$ ) resulting from the rotor-stator interaction are not the dominant components of the pressure fluctuation. Instead, the impeller rotating frequency $\left(f_{\mathrm{n}}\right)$ is very strong and becomes the dominant component, indicating that the flow inside the intake duct is periodic with the same frequency as the impeller (i.e., $f_{\mathrm{n}}$ ).

Furthermore, the normalized helicity $H_{\mathrm{n}}$ is introduced to capture the periodic flows and vortex motions with the definition in Equation (7) (Roth, 2000).

$H_{\mathrm{n}}=\frac{V \bullet(\nabla \times V)}{|V||\nabla \times V|}$ 


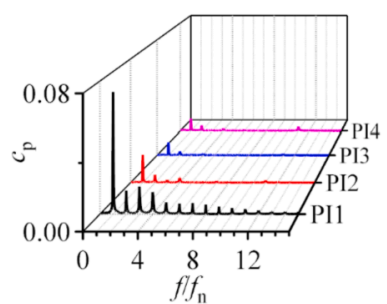

(a) 34 knot

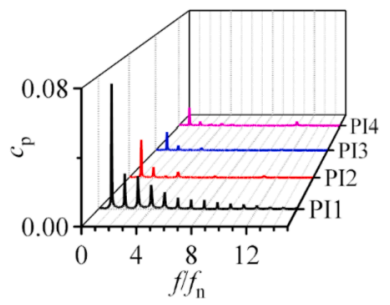

(b) 40 knot

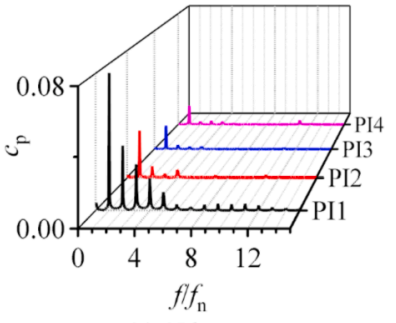

(c) 45 knot

Fig. 15. Pressure fluctuations of four monitoring points in the waterjet impeller at the cruising speed of (a) 34 knot, (b) 40 knot and (c) 45 knot.

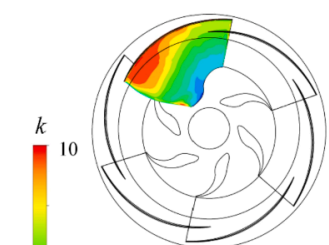

(a)

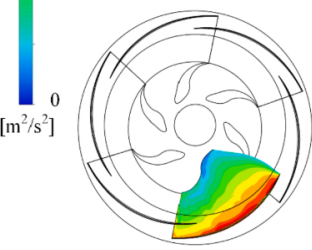

(d)

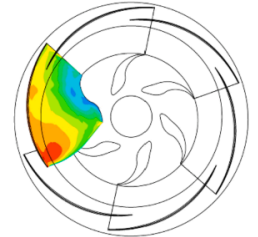

(b)

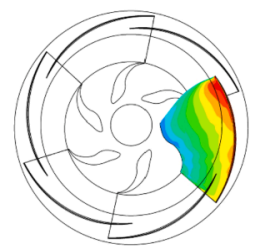

(e)

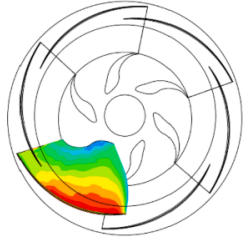

(c)

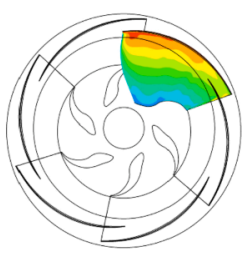

(f)
Fig. 16. Instantaneous distributions of the turbulent kinetic energy $k$ at the cruising speed of 45 knot: (a) $t_{0}$; (b) $t_{0}+1 / 6 T$; (c) $t_{0}+2 / 6 T$; (d) $t_{0}+3 / 6 T$; (e) $t_{0}+4 / 6 T$; (f) $t_{0}+5 / 6 T$.

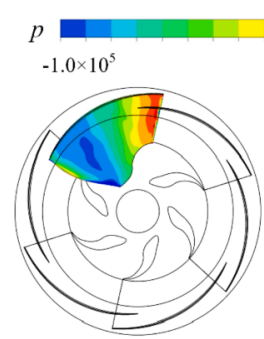

(a)

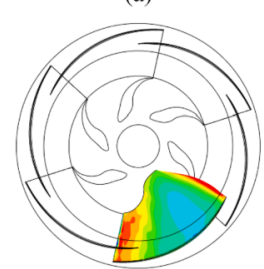

(d)
$[\mathrm{Pa}]$

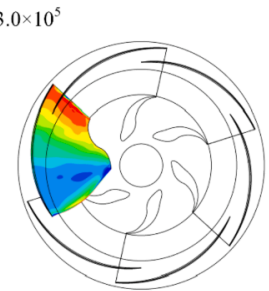

(b)

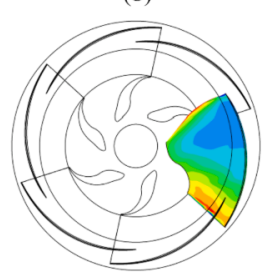

(e)

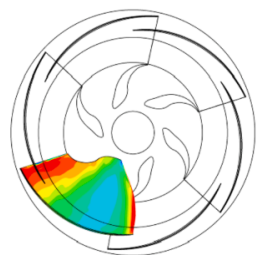

(c)

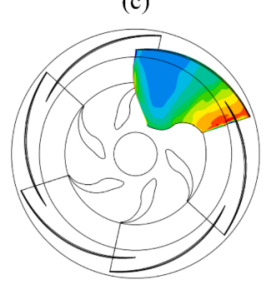

(f)
Fig. 17. Instantaneous distributions of the pressure on the blade at the cruising speed of 45 knot: (a) $t_{0}$; (b) $t_{0}+1 / 6 T$; (c) $t_{0}+2 / 6 T$; (d) $t_{0}+3 / 6 T$; (e) $t_{0}+4 / 6 T$; (f) $t_{0}+5 / 6 T$.

where $V$ is the velocity, $\nabla \times V$ is the vorticity. The normalized helicity $H_{\mathrm{n}}$ varies from -1 to +1 . $H_{\mathrm{n}}>0$ means the vortex motion is promoted and $H_{\mathrm{n}}<0$ indicates the vortex is suppressed.

Fig. 20 shows the instantaneous $H_{\mathrm{n}}$ distributions at the mid-plane of the intake duct during one impeller rotation cycle. At the cruising speed of 34 knot and $40 \mathrm{knot}$, the helicity distribution shows almost no variation at the mid-plane during one impeller rotation cycle as depicted in Fig. 20(a) and (b). At the speed of 34 knot, the normalized helicity

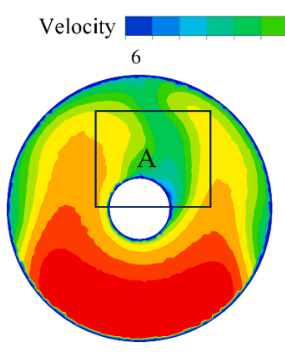

(a)

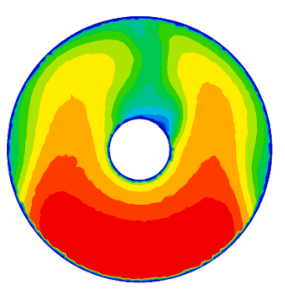

(d)

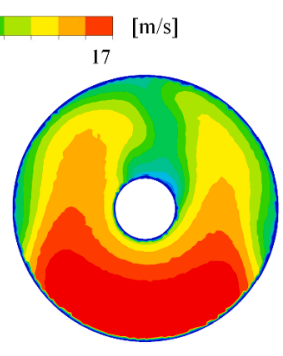

(b)

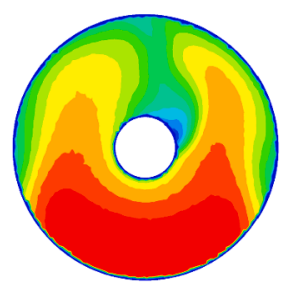

(e)

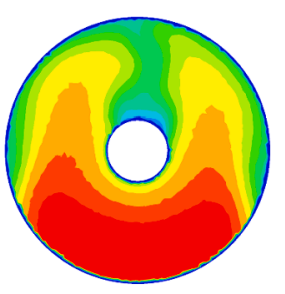

(c)

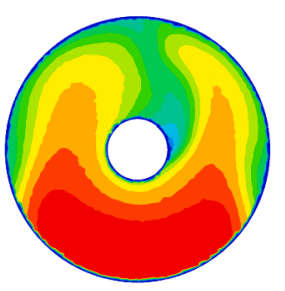

(f)
Fig. 18. Instantaneous distributions of the velocity at the Plane 1 at the cruising speed of 45 knot: (a) $t_{0}$; (b) $t_{0}+1 / 6 T$; (c) $t_{0}+2 / 6 T$; (d) $t_{0}+3 / 6 T$; (e) $t_{0}+4 / 6 T$; (f) $t_{0}+5 / 6 T$.

exhibits negative values in most regions, especially above the rotating shaft, indicating the vortex is suppressed in the intake duct. When the cruising speed is increased to $40 \mathrm{knot}$, the positive helicity exhibits near the upper side of the duct, so the vortex structures are enhanced. Besides, at the speed of 45 knot in Fig. 20 (c), the helicity distribution becomes disordered together with several positive and negative helicity regions above the rotating shaft, and thus the internal flow gets more complex, resulting in a larger nonuniformity at the Plane 1 in Fig. 12. It is observed that the positive helicity region (marked with a red dashed square) is not only enlarged in time but also moves downstream in space. Note that the helicity distribution is the same at $t=T_{4}$ with that at $t=T_{0}$, demonstrating that the positive helicity moves periodically and the frequency corresponds to the impeller rotating frequency $\left(f_{n}\right)$, which is consistent with the illustrations in Fig. 19 (c).

\subsubsection{Diffuser}

Four points are selected at the diffuser blade to analyse the pressure fluctuations by performing Fast Fourier Transform for the 15-cycle data. Fig. 21 shows the pressure frequency spectrums of the four monitoring points at various cruising speeds. For points PV1 and PV2, the dominant frequency is sixth impeller rotating frequency $\left(6 f_{\mathrm{n}}\right)$. It is because these two points are near the rotating impeller and effected by the rotor-stator interaction. Along with the fluid moving downstream, the effect of the rotor-stator interaction becomes weaker, and thus the pressure amplitude of point PV2 is much smaller than the pressure amplitude of point PV1. When the fluid sequentially propagates to point PV3 and PV4, the frequency spectrums present very complex signals. At the cruising speeds of 34 knot and 40 knot, the dominant frequency is $1.5 f_{n}$, and the 


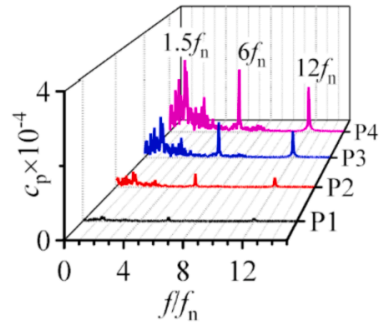

(a) 34 knot

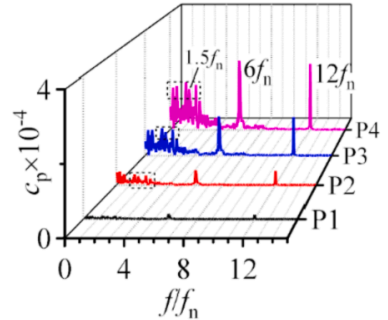

(b) 40 knot

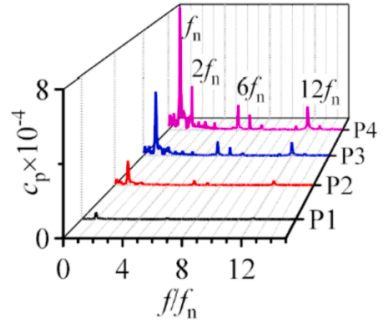

(c) 45 knot

Fig. 19. Pressure fluctuations of point P1, P2, P3, P4 inside the intake duct at the cruising speed of (a) 34 knot, (b) 40 knot and (c) 45 knot.

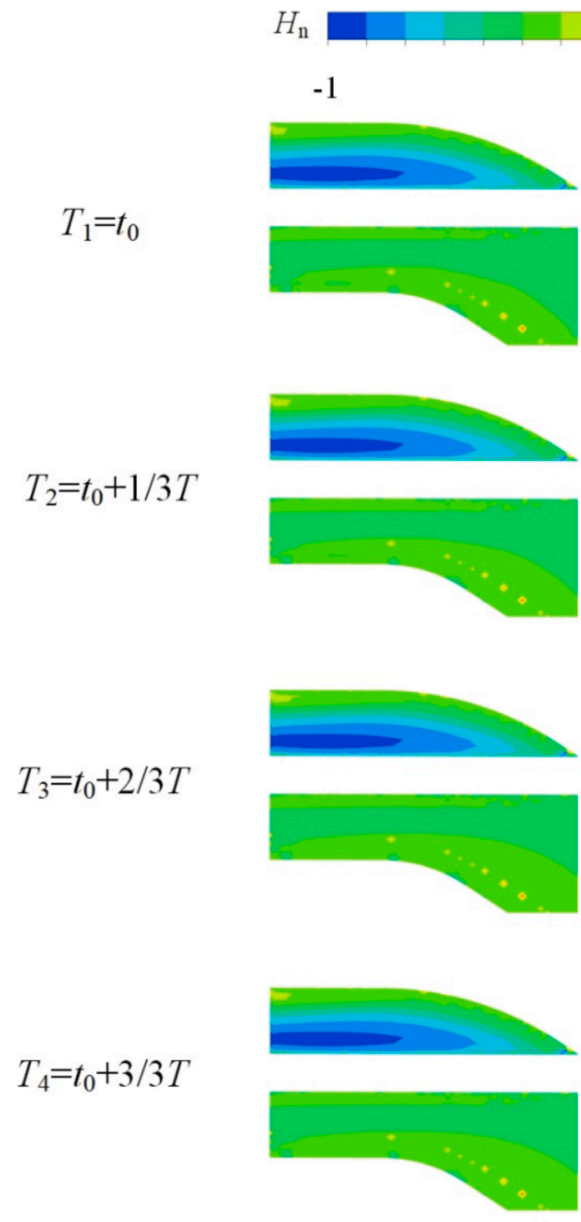

(a)
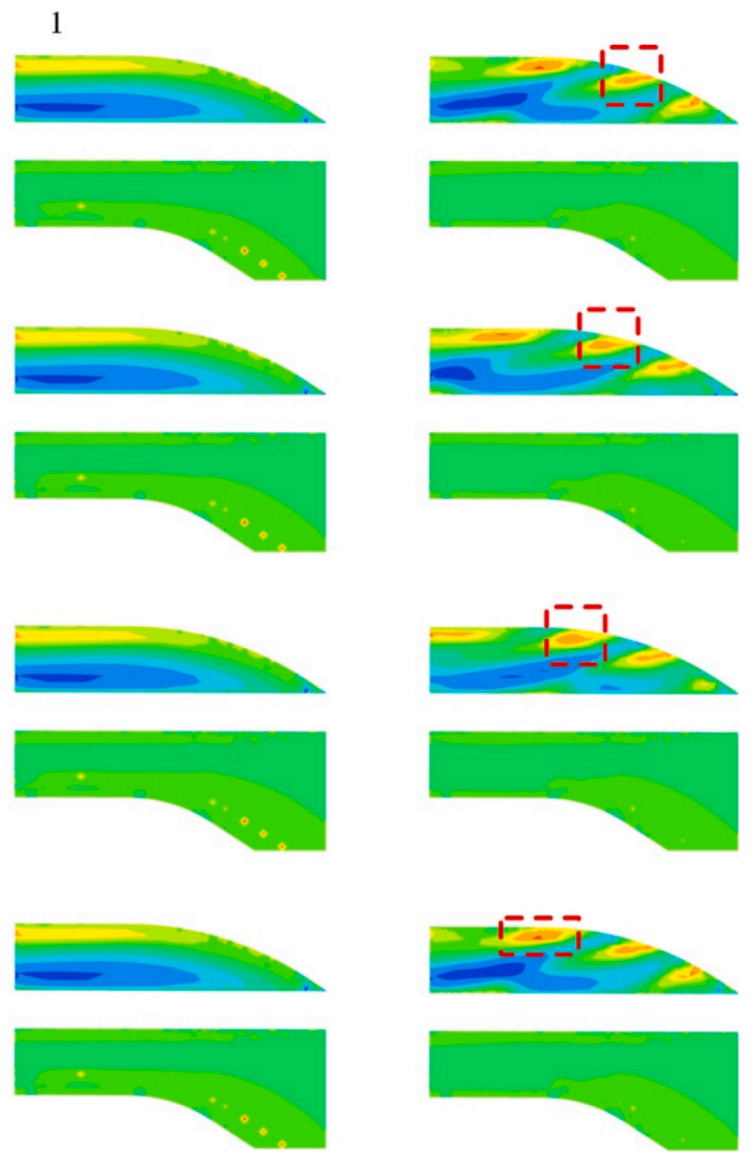

(b)

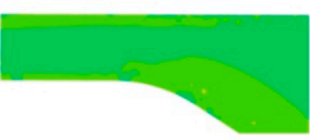

(c)

Fig. 20. Instantaneous $H_{\mathrm{n}}$ distributions at the mid-plane of the intake duct at three cruising speeds: (a) 34 knot, (b) 40 knot and (c) 45 knot.

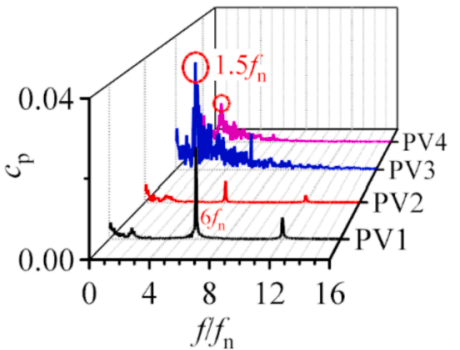

(a) $34 \mathrm{knot}$

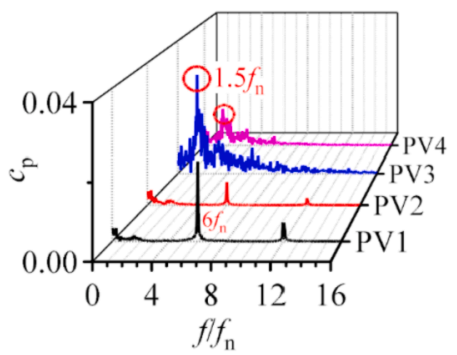

(b) $40 \mathrm{knot}$

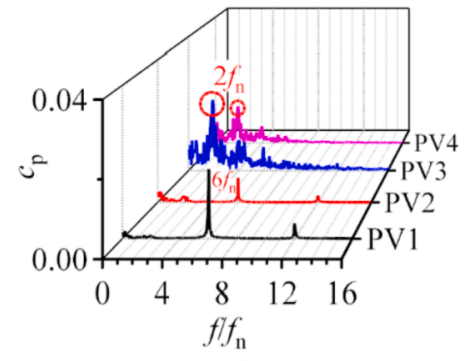

(c) $45 \mathrm{knot}$

Fig. 21. Pressure fluctuations of four monitoring points in the diffuser at the cruising speed of (a) 34 knot, (b) 40 knot and (c) 45 knot. 
corresponding amplitude is much smaller for point PV4 when compared with that of point PV3. This dominant frequency is resulted from the vortex evolution in the diffuser passage, the vortex is of great strength at point PV3 and then gradually sheds off at point PV4, which will be further illustrated in Fig. 22.

The frequency spectrums are similarly distributed for 34 knot and 40 knot, and the dominant frequency is $1.5 f_{\mathrm{n}}$. In contrast, the dominant frequency is $2 f_{\mathrm{n}}$ when the cruising speed reaches 45 knot. Based on the analyses of the flow characteristics in the intake duct as shown in Figs. 19 and 20, the vortex evolution is periodic inside the intake duct at 45 knot with the component of $2 f_{n}$, this is attributed to the upstream propagation of the pressure pulsation at point PV3 and will be discussed in section 3.3.4.

In order to clarify the demonstration above, the Q-criterion (Hunt et al., 1988) is adopted to show the unsteady characteristics of the vortex structures in the diffuser passage. It is defined in Equation (8), where $\nabla \mathbf{V}$ is the velocity gradient tensor and it can be decomposed into two parts, $i$. e., the symmetric part $\mathbf{A}$ and the anti-symmetric part $\mathbf{B}, a$ and $b$ are the squares of the Frobenius norm of $\mathbf{A}$ and $\mathbf{B}$. The $Q$ criterion is the difference of the symmetric part $\mathbf{A}$ and the anti-symmetric part $\mathbf{B}$, which is also the second invariant of the velocity gradient tensor.

$$
\begin{aligned}
& \nabla \mathbf{V}=\frac{1}{2}\left(\nabla \mathbf{V}+\nabla \mathbf{V}^{T}\right)+\frac{1}{2}\left(\nabla \mathbf{V}-\nabla \mathbf{V}^{T}\right)=\mathbf{A}+\mathbf{B} \\
& a=\operatorname{trace}\left(\mathbf{A}^{T} \mathbf{A}\right), \quad b=\operatorname{trace}\left(\mathbf{B}^{T} \mathbf{B}\right) \\
& Q=\frac{1}{2}(b-a)
\end{aligned}
$$

Fig. 22 shows the vortex evolution at the cruising speed of 40 knot by using the iso-surface of $Q=3.5 \times 10^{6} \mathrm{~s}^{-2}$. At $t=t_{0}$, two vortex structures can be observed in the diffuser passage. Vortex A is close to the blade leading edge (LE), and vortex B is in the middle of the blade. At $t=t_{0}+1$ / $6 T$, vortex $A$ is enlarged and moving downstream with vortex B shedding off from the blade trailing edge (TE). At the same time, there is a new vortex $\mathrm{B}^{\prime}$ generating at the blade leading edge (LE) with a small structure. At $t=t_{0}+2 / 6 T$, vortex A moves to the blade trailing edge and then vanishes at $t=t_{0}+3 / 6 T$ as shown in Fig. 22 (d), while vortex B' gradually develops with a large structure and continues to transport at the middle of the blade as shown in Fig. 22 (d). It is noted that the vortex structures at $t=t_{0}+3 / 6 T$ are distributed similarly with that at $t=t_{0}$. Therefore, the vortex evolution cycle is $2 / 6 \mathrm{~T}$ in diffuser passage, and this would lead to corresponding pressure pulsation phenomenon. The frequency for the vortex evolution is approximately $1.5 f_{n}$, which is consistent with the component of the pressure spectrum analysis in Fig. 21 (b).

The vorticity transport equation is used to give a deep insight into the mechanism of the vortices movement in the diffuser passage.

$D \frac{\vec{\omega}}{D t}=(\vec{\omega} \bullet \nabla) \vec{V}-\vec{\omega}(\nabla \bullet \vec{V})+\frac{\nabla \rho \times \nabla p}{\rho^{2}}+\left(\nu+\nu_{t}\right) \nabla^{2} \vec{\omega}$

In Equation (9), the first term on the right-hand-side (RHS) is the vortex stretching term by the velocity gradients, which represents the stretching and tilting of a vortex. The second term on the RHS is the vortex dilation due to volumetric expansion/contraction. The third term on the RHS is the baroclinic torque due to the misaligned between density and pressure gradients. Note that the second and third terms are zero in present study since the fluid here is assumed to be incompressible and there is no density variation in the flow field. The last term on the RHS is the viscous dissipation due to viscous diffusion of the vorticity. The last term has a much smaller effect on the vorticity transport than the other terms in high Reynolds number flow, and thus it can be neglected (Huang et al., 2015a; Ji et al., 2015; Ye et al., 2019). Therefore, only the vortex stretching term is discussed in present study.

A section plane is selected through point PV3 since the pressure amplitude at point PV3 is the strongest as shown in Fig. 21. Fig. 23 presents the transient contours of the vortex stretching term on the diffuser blade, the hub, and the cross-section plane. It is seen that the vortex stretching term on the blade and the hub is much larger than that distributed on the section plane, since the vorticity magnitude is larger on the walls and it quickly diminishes as the normal distance from the wall increases. With combined analyses from Fig. 22, large vortex stretching regions are located at the diffuser inlet, at the diffuser blade and close to the hub, which corresponds to the positions of vortex A and vortex B at $t=t_{0}$. At $t_{0}+1 / 6 T$, the high-value region on the section plane is enlarged while the high-value region on the hub becomes smaller, indicating the vortex structure would lead to strong deformation when passing through the section plane and the vortex strength decreases dramatically with a smaller vortex $\mathrm{B}^{\prime}$ generated at the diffuser inlet. With the developing of vortex B' and the moving downstream of vortex A at $t_{0}+2 / 6 T$, the stretching term becomes strong on the hub and drops a lot at the section plane. Meanwhile, the high-value region at the diffuser blade is strongly distributed at the blade LE and the interface of vortex structures as shown in Fig. 22,indicating that the distribution of the vortex stretching term are consistent with the vortex location.

Based on those vortex behaviours, the unsteady vortex evolution in the diffuser would make great contributions to the pressure pulsation at point PV3 and PV4. The vortex would generate at the diffuser inlet, developing and moving downstream, and disappear at the diffuser exit. The distribution of the vortex stretching term are consistent with the vortex location, and it is of great significance to the vorticity transport.

\subsubsection{Pressure fluctuation propagation}

The propagations of the pressure fluctuation are shown in Fig. 24 with the dominant frequency listed in Table 3 . The pressure fluctuates intensively in the impeller with the dominant frequency of the impeller rotating frequency $\left(f_{n}\right)$, and the amplitude of the pressure pulsation gradually decreases in the streamwise direction with the largest amplitude near the impeller inlet. Note that the amplitude of the dominant frequency in the impeller $\left(f_{\mathrm{n}}\right)$ is above 0.01 . In contrast, the amplitude of $1.5 f_{\mathrm{n}}$ in the impeller at $40 \mathrm{knot}$ is around $10^{-4}$ except point PI1, the amplitude of $2 f_{\mathrm{n}}$ in the impeller at 45 knot is around $10^{-3}$, indicating that the amplitude of the low-frequency component in the impeller is about two orders of magnitude smaller. Therefore, only the dominant frequency spectrum is depicted in Fig. 15. Basically, the pressure vibration in the intake duct is very small, and only the $f_{\mathrm{n}}$ component at high speed (i.e. 45 knot) seems dominant.

For the cruising speeds of 34 knot and 40 knot, the dominant frequency of the pressure fluctuation is the sixth impeller rotating frequency $\left(6 f_{n}\right)$ in the intake duct and near the diffuser inlet due to the effect of the rotor-stator interaction, while the dominant frequency is $1.5 f_{\mathrm{n}}$ near the diffuser outlet which is induced by the transient vortex evolution in the diffuser passage, as depicted in Figs. 22 and 23. As

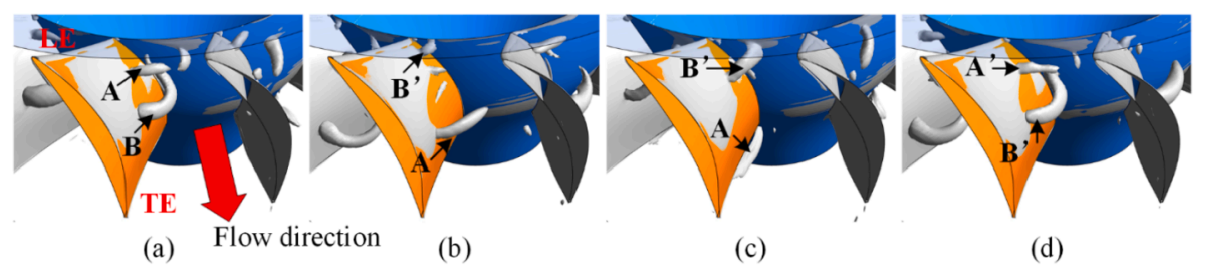

Fig. 22. Vortex development at several instants at the cruising speed of 40 knot: (a) $t_{0}$; (b) $t_{0}+1 / 6 T$; (c) $t_{0}+2 / 6 T$; (d) $t_{0}+3 / 6 T$. 

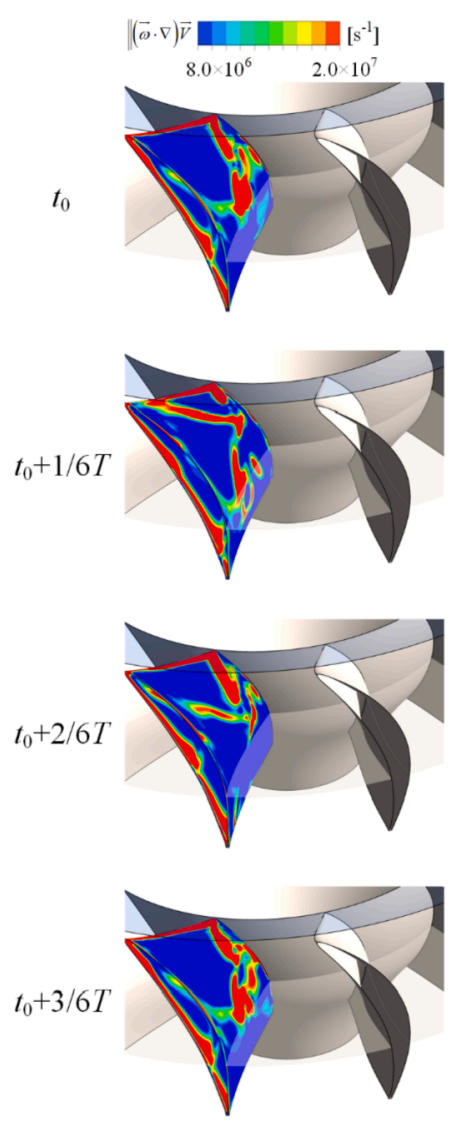

(a) diffuser blade
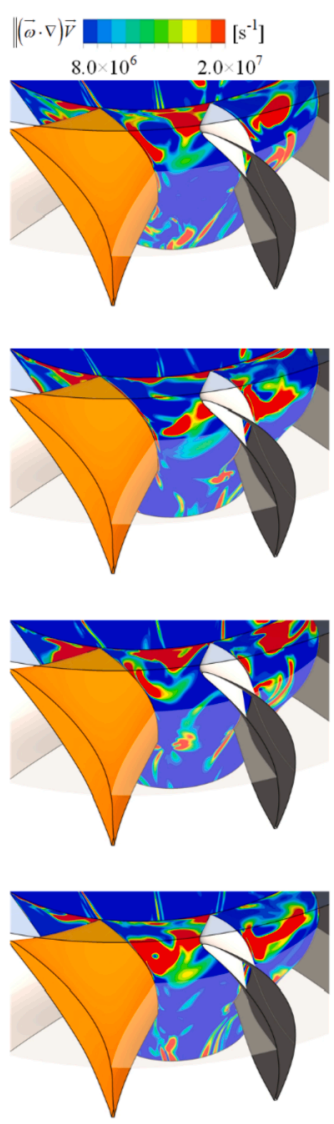

(b) hub
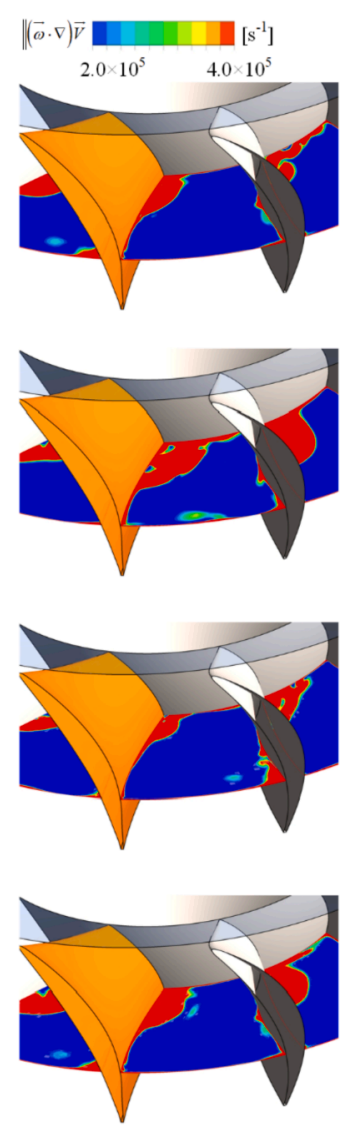

(c) cross-section plane

Fig. 23. Instantaneous contours of the vortex stretching term at 40 knot: (a) $t_{0}$; (b) $t_{0}+1 / 6 T$; (c) $t_{0}+2 / 6 T$; (d) $t_{0}+3 / 6 T$.

shown in Fig. 24 (a), this component of $1.5 f_{\mathrm{n}}$ from the vortex is also observed in the impeller and the intake duct.

When the cruising speed reaches $45 \mathrm{knot}$, the rotor-stator interaction still makes contribution to the pressure pulsations in the diffuser inlet with the dominant frequency of the sixth blade passing frequencies $\left(6 f_{n}\right)$, but the dominant frequency is $2 f_{\mathrm{n}}$ near the diffuser outlet caused by the unsteady vortex development. As depicted in Fig. 24 (b), the signal of $2 f_{\mathrm{n}}$ is also captured in the upstream, i.e. the impeller and the intake duct, while the amplitude of the pressure fluctuation decreases gradually. However, due to the effect of the impeller rotation, the flow inside the duct presents a periodic vortex motion called as prewhirl flow in Fig. 20, and the dominant frequency herein is the impeller rotating frequency $\left(f_{\mathrm{n}}\right)$.

\section{Conclusions}

In this paper, the unsteady flows in a waterjet propulsion system are investigated at various cruising speeds by analyzing the propulsion performance, time-averaged internal flows, pressure fluctuations and vortex evolutions. Conclusions are drawn as follows:

(1) The numerical methodology used in this study includes the Reynolds-Averaged Navier-Stokes (RANS) equation with the SST $k-\omega$ turbulence model, a sliding mesh technique. The hydrodynamic performance predicted by the present numerical approach is in good agreement with the available experimental data.

(2) In the intake duct, the augment of the cruising speed would lead to an increase in the nonuniformity and a reduction in the perpendicularity together with a slight variation in the hydraulic efficiency, indicating a worse inflow condition provided for the waterjet pump. Besides, the efficiency of the waterjet pump decreases by $0.25 \%$ when the cruising speed increases from $34 \mathrm{knot}$ to 45 knot.

(3) For the time-averaged flows, the pressure together with the nonuniformity increases when the flow passes through the impeller and then it drops after the diffuser. The pressure distribution is unsymmetrical at the impeller inlet plane due to the rotation effect of the impeller. The alternating presence of high pressure and low pressure is observed at the inlet and outlet planes of the diffuser with higher pressure at the diffuser inlet, and the pressure alternating phenomena becomes distinct along with the increase in the cruising speed.

(4) The pressure fluctuates intensively in the impeller with the dominant frequency of the first impeller rotating frequency $\left(f_{n}\right)$, and it gradually decreases in the streamwise direction with the largest amplitude near the impeller inlet. The rotor-stator interaction would cause a high-frequency component of sixth impeller rotating frequency $\left(6 f_{\mathrm{n}}\right)$ in the intake duct and near the diffuser inlet. Besides, a component having the frequency of $1.5 f_{\mathrm{n}}$ at 34 knot and 40 knot, and $2 f_{\mathrm{n}}$ at 45 knot is generated by the unsteady vortex evolution in the diffuser passage, which is also captured in the upstream intake duct and impeller. Analyses based on the vorticity transport equation demonstrate that the vortex stretching term makes a great contribution to the vorticity distribution and evolution in the diffuser.

(5) At the cruising speed of $45 \mathrm{knot}$, the flow inside the duct is strongly affected by the impeller rotation and presents a periodic prewhirl motion with the dominant frequency of the first impeller rotating frequency $\left(f_{\mathrm{n}}\right)$. 


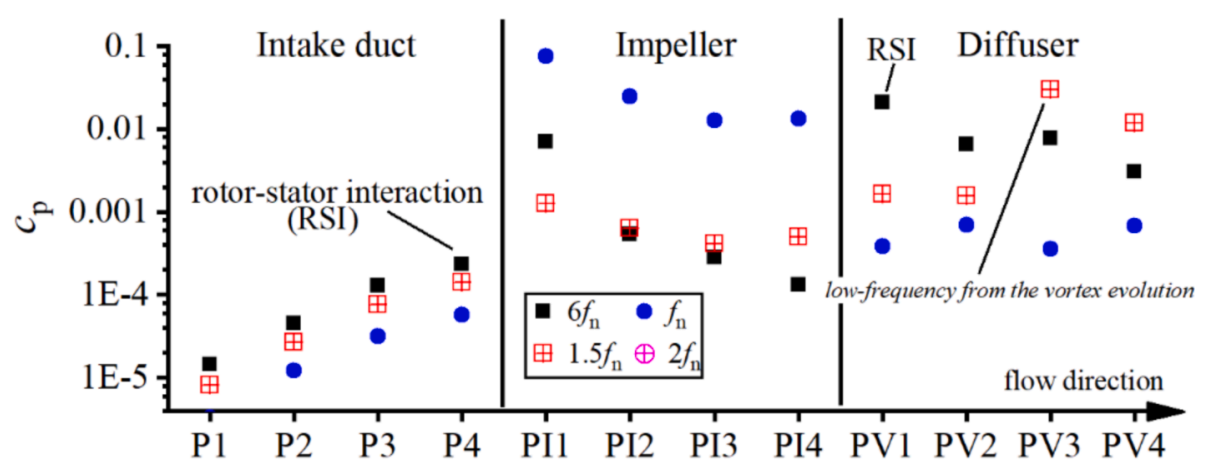

(a) 40 knot

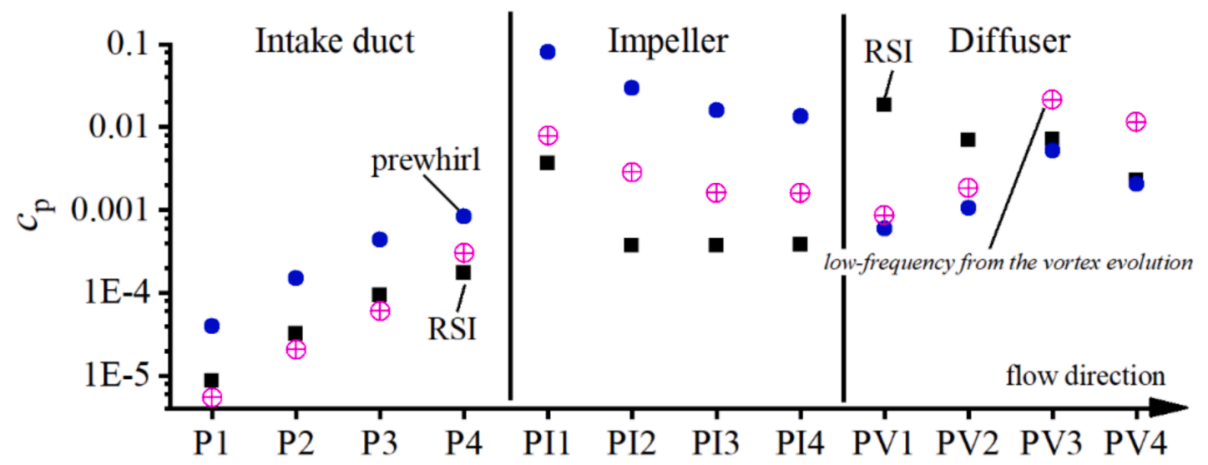

(b) 45 knot

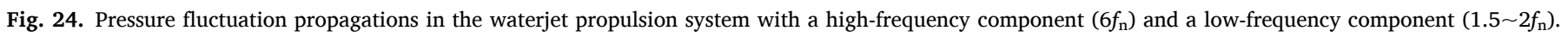

Table 3

Dominant frequency of the pressure fluctuation in the waterjet propulsion system.

\begin{tabular}{lllll}
\hline Domain & & 34 knot & 40 knot & 45 knot \\
\hline Intake duct & & $6 f_{\mathrm{n}}$ & $6 f_{\mathrm{n}}\left(1.5 f_{\mathrm{n}}\right)$ & $f_{\mathrm{n}}$ \\
Impeller & & $f_{\mathrm{n}}$ & $f_{\mathrm{n}}$ & $f_{\mathrm{n}}$ \\
Diffuser & PV1, PV2 & $6 f_{\mathrm{n}}$ & $6 f_{\mathrm{n}}$ & $6 f_{\mathrm{n}}$ \\
& PV3, PV4 & $1.5 f_{\mathrm{n}}$ & $1.5 f_{\mathrm{n}}$ & $2 f_{\mathrm{n}}$ \\
\hline
\end{tabular}

\section{Author contributions section}

Renfang Huang: Conceptualization, Writing - Original Draft, Validation.

Weixiang Ye: Data Curation.

Yuanxing Dai: Methodology.

Xianwu Luo: Writing - Review \& Editing.
Yiwei Wang: Funding acquisition.

Tezhuan Du: Formal analysis.

Chenguang Huang: Supervision.

\section{Declaration of competing interest}

The authors declare that they have no known competing financial interests or personal relationships that could have appeared to influence the work reported in this paper.

\section{Acknowledgment}

This work was financially supported by the National Key R\&D Program of China (2017YFC1404200, 2018YFB0606101) and National Natural Science Foundation of China (Grant Nos 51776102 , 11772340 and 11672315) and the Science and Technologyon Water Jet Propulsion Laboratory (Grant No. 6142223190101).

\section{Nomenclature}

BEM Boundary Element Model

DOF Degree-of-Freedom

ITTC International Towing Tank Conference

LE Leading Edge

TE Trailing Edge

PS Pressure Surface

RANS Reynolds-Averaged Navier-Stokes

RMS Root Mean Square

RHS Right-Hand-Side

SAS Scale-Adaptive Simulation

SPIV Stereo Particle Image Velocimetry

SS Suction Surface 
TLV

UDS

VOF

$c_{\mathrm{p}}$
$D$

Duct diameter, $D=244 \mathrm{~mm}$

$E_{\text {in }} \quad$ Total energy at the inlet plane positioned one impeller diameter forward of the ramp tangent point

$E_{\text {out }} \quad$ Total energy at the outlet plane

$f_{\mathrm{n}} \quad$ Impeller rotating frequency

$H_{\mathrm{n}} \quad$ Normalized helicity

$k \quad$ Turbulent kinetic energy

$n \quad$ Rotation speed

$p_{\text {in }}, p_{\text {out }} \quad$ Static pressure at the inlet plane and outlet plane, respectively

$p_{\mathrm{r}} \quad$ Reference pressure

$p_{i} \quad$ Instantaneous value of static pressure at a monitoring point

$p_{0} \quad$ Averaged pressure at the impeller inlet plane

$R_{2} \quad$ Radius of the blade tip at the exit

$V_{2} \quad$ Circumferential velocity at the impeller exit, $V_{2}=2 \pi n R_{2} / 60$

$V_{\mathrm{a}}, \overline{V_{\mathrm{a}}} \quad V_{\mathrm{t}}$ The axial velocity, the averaged axial velocity and the tangential velocity at the outlet plane, respectively

$V_{\text {in }}, V_{\text {out }}$ Velocity at the inlet plane and outlet plane, respectively

$V_{\mathrm{wt}} \quad$ Local velocity at the inlet plane of the water tank with a distance of $y_{\text {rel }}$ from the hull

$V_{\mathrm{s}} \quad$ Ship cruising speed

$\delta \quad$ Thickness of the hull boundary layer

$L_{\text {in }} \quad$ The distance from the domain inlet plane to the inlet, $L_{\text {in }}=25 D$

Re Reynolds number, $\operatorname{Re}=V_{\mathrm{s}} L_{\mathrm{in}} / v$

$v \quad$ Fluid kinematic viscosity

Q Flow rate

$Q$ criterion The second invariant of the velocity gradient tensor

$\eta_{\text {duct }} \quad$ Hydraulic efficiency

$\xi \quad$ Outflow nonuniformity

$\varphi_{\mathrm{p}} \quad$ Perpendicularity

$\rho \quad$ Fluid density

\section{References}

Acosta, A., 1958. An experimental study of cavitating inducers. ONR/ACR-38. In: Proceedings of the Second Symposium on Naval Hydrodynamics, pp. 537-557.

Altosole, M., Benvenuto, G., Figari, M., Campora, U., 2012. Dimensionless numerical approaches for the performance prediction of marine waterjet propulsion units. Int J. Rotating Mach. 12, 2012.

Bardina, J.E., Huang, P.G., Coakley, T.J., 1997. Turbulence Modeling Validation, Testing, and Development. NASA TM 110446.

Bulten, N., van Esch, B., 2005. Review of thrust prediction method based on momentum balance for ducted propellers and waterjets. In: ASME 2005 Fluids Engineering Division Summer Meeting. American Society of Mechanical Engineers, pp. 1621-1629.

Bulten, N.W.H., 2006. Numerical Analysis of a Waterjet Propulsion System. Technische Universiteit Eindhoven, Netherlands.

Cao, P., Wang, Y., Kang, C., Li, G., Zhang, X., 2017. Investigation of the role of nonuniform suction flow in the performance of water-jet pump. Ocean. Eng. 140, 258-269.

Cheng, H.Y., Bai, X.R., Long, X.P., Ji, B., Peng, X.X., Farhat, M., 2020. Large eddy simulation of the tip-leakage cavitating flow with an insight on how cavitation influences vorticity and turbulence. Appl. Math. Model. 77, 788-809.

Delaney, K., Donnely, M., Elbert, M., Fry, D., 2009. Use of RANS for waterjet analysis of a high-speed sealift concept vessel. In: 1st International Symposium on Marine Propulsors, Trondheim, Norway.

Ding, J., Wang, Y., 2010. Research on flow loss of inlet duct of marine waterjets. Journal of Shanghai Jiaotong University (Science) 15 (2), 158-162.

Eça, L., Hoekstra, M., Roache, P., 2005. Verification of calculations: an overview of the Lisbon workshop. In: 23rd AIAA Applied Aerodynamics Conference, p. 4728.

Eça, L., Hoekstra, M., Roache, P., 2007. Verification of calculations: an overview of the 2nd lisbon workshop. In: 18th AIAA Computational Fluid Dynamics Conference, p. 4089.

Eslamdoost, A., 2012. Investigations of Waterjet/hull Interaction Effects. Department of Shipping and Marine Technology Chalmers University of Technology, Gothenburg, Sweden.

Eslamdoost, A., 2014. The Hydrodynamics of Waterjet/Hull Interaction. Department of Shipping and Marine Technology Chalmers University of Technology, Gothenburg, Sweden.

Eslamdoost, A., Larsson, L., Bensow, R., 2014. A pressure jump method for modeling waterjet/hull interaction. Ocean. Eng. 88, 120-130.

Eslamdoost, A., Larsson, L., Bensow, R., 2018. Analysis of the thrust deduction in waterjet propulsion - the Froude number dependence. Ocean. Eng. 152, 100-112.
Eslamdoost, A., Vikström, M., 2019. A body-force model for waterjet pump simulation. Appl. Ocean Res. 90, 101832

Forest, S., Moreau, S., Desrochers, A., Asme, 2012. 3D unsteady simulation of a planing hull propelled by a waterjet pump system. Proceedings of the Asme Fluids Engineering Division Summer Meeting 179-189, 2012, Vol 1, Pts a and B, Symposia. Amer Soc Mechanical Engineers, New York.

Gao, H., Lin, W., Du, Z., 2008. Numerical flow and performance analysis of a water-jet axial flow pump. Ocean. Eng. 35 (16), 1604-1614.

Gong, J., Guo, C., Wang, C., Wu, T., Song, K., 2019. Analysis of waterjet-hull interaction and its impact on the propulsion performance of a four-waterjet-propelled ship. Ocean. Eng. 180, 211-222.

Griffith-Jones, G.J., 1994. Investigation of Incompressible Flow through an Intake Duct with Applications to Waterjet Propulsion. Mechanical Engineering. University of Canterbury.

Guo, Q., Huang, X., Qiu, B., 2019. Numerical investigation of the blade tip leakage vortex cavitation in a waterjet pump. Ocean. Eng. 187, 106170.

Hino, T., Ohashi, K., 2009. Numerical simulation of flow around a waterjet propelled ship. In: Proceedings of the 1st International Symposium on Marine Propulsors. Trondheim, Norway.

Hu, F., Wu, P., Wu, D., Wang, L., 2014. Numerical study on the stall behavior of a water jet mixed-flow pump. J. Mar. Sci. Technol. 19 (4), 438-449.

Huang, R., Dai, Y., Luo, X., Wang, Y., Huang, C., 2019a. Multi-objective optimization of the flush-type intake duct for a waterjet propulsion system. Ocean. Eng. 187, 106172.

Huang, R., Ji, B., Luo, X., Zhai, Z., Zhou, J., 2015a. Numerical investigation of cavitationvortex interaction in a mixed-flow waterjet pump. J. Mech. Sci. Technol. 29 (9), 3707-3716.

Huang, R., Luo, X., Ji, B., Wang, P., Yu, A., Zhai, Z., Zhou, J., 2015b. Multi-objective optimization of a mixed-flow pump impeller using modified NSGA-II algorithm. Sci. China Technol. Sci. 1-9.

Huang, R., Shao, S., Arndt, E.A.R., Luo, X., Wang, Y., Hong, J., et al., 2020. Numerical Study of the Behaviors of Ventilated Supercavities in a Periodic Gust Flow. Journal of fluids engineering 142 (6), 61403. https://doi.org/10.1115/1.4046110.

Huang, R., Yu, A., Ji, B., Zhou, J., Zhai, Z., Luo, X., 2019b. Cavitating flow features in a water-jet pump under different upstream conditions. In: 16th International Symposium on Transport Phenomena and Dynamics of Rotating Machinery, ISROMAC 2016. International Symposium on Transport Phenomena and Dynamics of Rotating Machinery. ISROMAC 2016.

Hunt, J.C.R., Wray, A.A., Moin, P., 1988. Eddies, Streams, and Convergence Zones in Turbulent Flows. Center for Turbulence Research, p. 193. Proceedings of the Summer Program. 
ITTC, 1999. ITTC - Recommended Procedures: CFD, Resistance and Flow Uncertainty Analysis in CFD Examples for Resistance and Flow.

Ji, B., Luo, X., Peng, X., Wu, Y., Xu, H., 2012. Numerical analysis of cavitation evolution and excited pressure fluctuation around a propeller in non-uniform wake. Int. J. Multiphas. Flow 43 (0), 13-21.

Ji, B., Luo, X.W., Arndt, R.E.A., Peng, X., Wu, Y., 2015. Large Eddy Simulation and theoretical investigations of the transient cavitating vortical flow structure around a NACA66 hydrofoil. Int. J. Multiphas. Flow 68 (0), 121-134.

Li, G., Wang, J., Zhou, J., 2017. Research on cavitation performance of axial flow water jet pumps with low specific speed. SHIPBUILDING OF CHINA 58 (04), 38-45.

Lindau, J.W., Pena, C., Baker, W.J., Dreyer, J.J., Moody, W.L., Kunz, R.F., Paterson, E.G., 2012. Modeling of cavitating flow through waterjet propulsors. Int. J. Rotating Mach. 13, 2012.

Liu, C., Wang, Y., Zhang, Z., Liu, J., 2010. Research on effect of different flow control volume on waterjet performance prediction. J. Ship Mech. (10), 1117-1121.

Liu, M., Tan, L., Cao, S., 2019. Dynamic mode decomposition of gas-liquid flow in a rotodynamic multiphase pump. Renew. Energy 139, 1159-1175.

Liu, Y., Tan, L., 2019. Spatial-temporal evolution of tip leakage vortex in a mixed-flow pump with tip clearance. J. Fluid Eng. 141 (8), 081302-081302-081311.

Luo, X., Ye, W., Huang, R., Wang, Y., Du, T., Huang, C., 2020. Numerical investigations of the energy performance and pressure fluctuations for a waterjet pump in a nonuniform inflow. Renewable Energy 153, 1042-1052. https://doi.org/10.1016/j. renene.2020.02.081.

Miorini, R.L., Wu, H.X., Katz, J., 2012. The internal structure of the tip leakage vortex within the rotor of an axial waterjet pump. Journal of Turbomachinery-Transactions of the Asme 134 (3), 12.

Miorini, R.L., Wu, H.X., Katz, J., Asme, 2010. The Internal Structure of the Tip Leakage Vortex within the Rotor of an Axial Waterjet Pump. Amer Soc Mechanical Engineers, New York.

Olsson, M., 2008. Numerical Investigation on the Cavitating Flow in a Waterjet Pump. Chalmers University of Technology, Sweden.

Park, W.-G., Yun, H.S., Chun, H.H., Kim, M.C., 2005a. Numerical flow simulation of flush type intake duct of waterjet. Ocean. Eng. 32 (17-18), 2107-2120.

Park, W.G., Jang, J.H., Chun, H.H., Kim, M.C., 2005b. Numerical flow and performance analysis of waterjet propulsion system. Ocean. Eng. 32 (14-15), 1740-1761.
Pouffary, B., Patella, R.F., Reboud, J.-L., Lambert, P.-A., 2008. Numerical simulation of 3D cavitating flows: analysis of cavitation head drop in turbomachinery. J. Fluid Eng. 130 (6), 061301-061301-061310.

Roache, P.J., 1993. A method for uniform reporting of grid refinement studies. ASMEPUBLICATIONS-FED 158, 109-109.

Roth, M., 2000. Automatic extraction of vortex core lines and other line type features for scientific visualization. Hartung-Gorre Verlag Konstanz.

Sauer, J., Schnerr, G., 2000. Unsteady cavitating flow-a new cavitation model based on a modified front capturing method and bubble dynamics. In: Proceedings of 2000 ASME Fluid Engineering Summer Conference, pp. 11-15.

Tan, D., Li, Y., Wilkes, I., Vagnoni, E., Miorini, R.L., Katz, J., 2015. Experimental investigation of the role of large scale cavitating vortical structures in performance breakdown of an axial waterjet pump. Journal of Fluids Engineering, Transactions of the ASME 137 (11).

Tan, D.Y., Miorini, R.L., Keller, J., Katz, J., 2012. Flow visualization using cavitation within blade passage of an axial waterjet pump rotor, ASME 2012 Fluids Engineering Division Summer Meeting. In: FEDSM 2012 Collocated with the ASME 2012 Heat Transfer Summer Conf. and the ASME 2012 10th International Conference on Nanochannels, Microchannels, and M, FEDSM 2012, PARTS A and B. Rio Grande, pp. 395-404.

van Esch, B., Cheng, L., 2011. Unstable operation of a mixed-flow pump and the influence of tip clearance. In: ASME-JSME-KSME 2011 Joint Fluids Engineering Conference. American Society of Mechanical Engineers, pp. 79-87.

Vanka, S.P., 1986. Block-implicit multigrid solution of Navier-Stokes equations in primitive variables. J. Comput. Phys. 65 (1), 138-158.

Wu, H., Miorini, R.L., Katz, J., 2011a. Measurements of the tip leakage vortex structures and turbulence in the meridional plane of an axial water-jet pump. Exp. Fluid 50 (4), 989-1003.

Wu, H., Tan, D., Miorini, R.L., Katz, J., 2011b. Three-dimensional flow structures and associated turbulence in the tip region of a waterjet pump rotor blade. Exp. Fluid 51 (6), 1721-1737.

Yang, Q.F., Wang, Y.S., Zhang, Z.H., 2014. Numerical prediction of the fluctuating noise source of waterjet in full scale. J. Mar. Sci. Technol. 19 (4), 510-527.

Ye, W., Luo, X., Huang, R., Jiang, Z., Li, X., Zhu, Z., 2019. Investigation of flow instability characteristics in a low specific speed centrifugal pump using a modified partially averaged Navier-Stokes model. Proc. IME J. Power Energy 233 (7), 834-848. 